\title{
ABSOLUTELY CONTINUOUS SPECTRUM FOR ONE-DIMENSIONAL SCHRÖDINGER OPERATORS WITH SLOWLY DECAYING POTENTIALS: SOME OPTIMAL RESULTS
}

\author{
MICHAEL CHRIST AND ALEXANDER KISELEV
}

\section{INTRODUCTION AND MAIN RESULTS}

In this paper, we study the stability of the absolutely continuous spectrum of one-dimensional Schrödinger operators under perturbations by slowly decaying potentials. Suppose that $H_{U}$ is a Schrödinger operator defined on $L^{2}(0, \infty)$ by the differential expression

$$
H_{U}=-\frac{d^{2}}{d x^{2}}+U(x)
$$

and some self-adjoint boundary condition at the origin. We assume that $U$ is some bounded function for which $H_{U}$ has absolutely continuous spectrum. The presence of the absolutely continuous spectrum has direct consequences for the physical properties of the quantum particle described by the operator $H_{U}$ (see, e.g. [23, 2]). If we perturb this operator by some decaying potential $V(x)$, the Weyl criterion implies that the essential spectra of the operators $H_{U}$ and $H_{U+V}$ coincide. We seek conditions on the rate of decay of $V(x)$ which ensure that the absolutely continuous spectrum of the unperturbed operator $H_{U}$ is also preserved.

This problem has a long history as one of the most natural questions in quantum mechanics, and we briefly recall the main results. It has long been known that if the perturbation $V(x)$ is absolutely integrable, then the absolutely continuous spectrum of the original operator is preserved. Until recently, little more was known concerning the preservation of the absolutely continuous spectrum of Schrödinger operators under decaying perturbations in the general situation.

Substantially more information is available in the case when $U(x)=0$. There has been much work on proving the absolute continuity of the spectrum for Schrödinger operators with potentials of slower decay, but satisfying some additional special assumptions. For example, by a result going back to Weidmann [31], if a potential $V$ may be represented as a sum of a function of bounded variation and an absolutely integrable function, then the spectrum of the operator $H_{V}$ on $\mathbb{R}^{+}=(0, \infty)$ is purely absolutely continuous. Many authors developed a scattering theory for longrange potentials whose derivatives satisfy certain bounds; see for example $[1,5,12]$.

Received by the editors June 30, 1997.

1991 Mathematics Subject Classification. Primary 34L40, 81Q05, 42B20; Secondary 81Q15, 42B25.

Key words and phrases. Schrödinger operator, absolutely continuous spectrum, a.e. convergence, decaying potential, WKB asymptotics, norm estimates.

The firts author's work was partially supported by NSF grant DMS96-23007.

The second author's work at MSRI was partially supported by NSF grant DMS 902140. 
These results hold in any dimension and the proofs involve approximating the scattering trajectories by solutions of the classical Hamilton-Jacobi equation. The weakest conditions on the long-range part of the potential under which the wave operators are known to exist are given in [12]. For potentials satisfying $|V(x)| \leq$ $C(1+|x|)^{-\frac{1}{2}-\epsilon}$, for instance, one can infer the existence of the wave operators if also $\left|D^{\alpha} V(x)\right| \leq C_{1}(1+|x|)^{-\frac{3}{2}-\epsilon}$ for every multi-index $\alpha$ with $|\alpha|=1$.

Another class of results describes spectral behavior of specific spherically symmetric (i.e. essentially one-dimensional) oscillating potentials, the typical example being $V(x)=x^{-\beta} \sin x^{\alpha}$ with $\alpha, \beta$ positive. Such potentials in general do not satisfy the derivative bounds needed for the method of the works cited above to be applicable. We mention the papers $[3,4,11,20,32]$ in which further references may be found. The spectrum of the operator $H_{V}$ for such potentials turns out to be absolutely continuous with perhaps some isolated embedded eigenvalues when $\alpha=1$. These potentials generalize the celebrated Wigner-von Neumann example [30]. Wigner and von Neumann were the first to discover an example with Coulomb type decay at infinity, i.e. $V(x)=O\left((1+|x|)^{-1}\right)$, whose spectrum is not purely absolutely continuous and has positive eigenvalues embedded in the absolutely continuous spectrum. Moreover, Naboko [22] and later Simon [26] found different constructions which show that for potentials decaying more slowly but arbitrarily close to a Coulomb rate, very striking spectral phenomena arise. Namely, for every function $C(x)$ tending monotonically to infinity as $x$ goes to infinity, no matter how slowly, there exists a potential $V(x)$ satisfying

$$
|V(x)| \leq \frac{C(x)}{1+|x|},
$$

for which the associated Schrödinger operator $H_{V}$ has a dense set of eigenvalues in $\mathbb{R}^{+}$.

A new general class of potentials preserving the absolutely continuous spectrum of the free Schrödinger operator was recently found by one of us in [13]. Namely, if the potential $V$ satisfies $|V(x)| \leq C(1+|x|)^{-\frac{3}{4}-\epsilon}$ with some $\epsilon>0$, with no additional assumptions, then the whole positive semi-axis $(0, \infty)$ is an essential support of the absolutely continuous part of the spectral measure. ${ }^{1}$ Of course, as the examples of Naboko and Simon show, a rich embedded singular spectrum may occur; however it is indeed embedded in the sense that there is an underlying absolutely continuous spectrum. One can describe the set where the singular part of the spectral measure might be supported in $\mathbb{R}^{+}$rather explicitly in terms of the properties of the Fourier transform of $x^{\frac{1}{4}} V(x)$ [13].

The result of [13] was further improved in [14], where a general criterion was established which implies the stability of the absolutely continuous spectrum of the operator $H_{U}$ under all perturbations $V(x)$ satisfying $|V(x)| \leq C(1+|x|)^{-\frac{2}{3}-\epsilon}$, under the auxiliary hypothesis that a certain operator, constructed from the generalized eigenfunctions of $H_{U}$, is bounded on $L^{2}\left(\mathbb{R}^{+}\right)$. In particular, it was shown that the absolutely continuous spectra of free and periodic one-dimensional Schrödinger operators are stable under all perturbations by potentials satisfying $|V(x)| \leq$ $C(1+|x|)^{-\frac{2}{3}-\epsilon}$. Later, this result for the case $U=0$ was also proved by S. Molchanov by a different method [21].

\footnotetext{
${ }^{1} E \subset \mathbb{R}$ is an essential support of the (projection-valued) measure $\mu$ if $\mu(X)>0$ for any $X \subset E$ of positive Lebesgue measure, and $\mu(\mathbb{R} \backslash E)=0$.
} 
On the other hand, work on random potentials by Kotani and Ushiroya [18] provides a bound for the best possible result that one could hope to prove. The results of [18] imply that (in the case $U=0$ ) there exist potentials $V(x)$ satisfying $V(x) \leq C(1+|x|)^{-\frac{1}{2}}$, for which $H_{V}$ has purely singular spectrum on $\mathbb{R}^{+}$. Indeed this happens almost surely for certain classes of random potentials. We remark that by combining methods used in recent works [16] and [17], one can show that the $(1+|x|)^{-\frac{1}{2}}$ rate of decay is also critical for perturbations of the periodic Schrödinger operators.

In this paper we establish results on the preservation of absolutely continuous spectrum for power decaying potentials in one dimension which are of an optimal nature. In particular, we prove

Theorem 1.1. Suppose that there exist $\epsilon>0$ and $p \leq 2$ so that $V(x)(1+|x|)^{\epsilon} \epsilon$ $L^{p}\left(\mathbb{R}^{+}\right)$. Then the whole positive semi-axis $\mathbb{R}^{+}=(0, \infty)$ is an essential support of the absolutely continuous part of the spectral measure of the operator $H_{V}$. Moreover, for almost every $\lambda \in \mathbb{R}^{+}$, there exist solutions $\phi_{\lambda}(x)$ and $\bar{\phi}_{\lambda}(x)$ of the generalized eigenfunction equation

$$
-y^{\prime \prime}+V(x) y=\lambda y
$$

with asymptotic behavior of pure WKB form in the main term:

$$
\phi_{\lambda}(x)=\exp \left(i \sqrt{\lambda} x-\frac{i}{2 \sqrt{\lambda}} \int_{0}^{x} V(t) d t\right)(1+o(1))
$$

as $x \rightarrow \infty$.

Corollary. If $V(x)=O(1+|x|)^{-r}$ for some $r>1 / 2$, then $(0, \infty)$ is an essential support of the absolutely continuous part of the spectral measure of $H_{V}$ on $L^{2}\left(\mathbb{R}^{+}\right)$.

We have a similar result for perturbations of periodic Schrödinger operators. Let $U$ be continuous and periodic, let $S=\bigcup_{n=0}^{\infty}\left(a_{n}, b_{n}\right)$ be the band spectrum of the unperturbed operator with potential $U$, and let $\theta(x, \lambda)$ be the Bloch functions for that operator.

Theorem 1.2. If the potential $V(x)$ is as in Theorem 1.1, then the set $S$ is an essential support of the absolutely continuous part of the spectral measure of the operator $H_{U+V}$. For a.e. $\lambda \in S$, there exist solutions $\psi_{\lambda}(x), \bar{\psi}_{\lambda}(x)$ of the equation

$$
-y^{\prime \prime}+(U(x)+V(x)) y=\lambda y
$$

with the asymptotic behavior

$$
\psi_{\lambda}(x)=\theta(x, \lambda) \exp \left(\frac{i}{2 \operatorname{Im}\left(\theta \bar{\theta}^{\prime}\right)} \int_{0}^{x} V(t)|\theta(t, \lambda)|^{2} d t\right)(1+o(1))
$$

as $x \rightarrow \infty$.

Both theorems will follow from a certain general criterion. Suppose that $H_{U}$ is an operator for which all solutions of the equation

$$
-y^{\prime \prime}+U(x) y=\lambda y
$$

are bounded for almost every $\lambda \in S$, where $S$ is a certain set of positive Lebesgue measure. It is known (see, e.g. [26, 29]) that under mild assumptions on $U$ (see Lemma 2.1) the set $S$ belongs to an essential support of the absolutely continuous 
part of the spectral measure. Let us pick a family of solutions $\theta(x, \lambda), \lambda \in S$, of the equation (3), such that $\theta(x, \lambda)$ are uniformly bounded over $S$ and $\theta(x, \lambda), \bar{\theta}(x, \lambda)$ are linearly independent for every $\lambda \in S$. It is easy to see that we can always find such a family. We have

Theorem 1.3. Suppose that the potential $V(x)$ is such that there exist $\epsilon>0$ and $p \leq 2$ so that $(1+|x|)^{\epsilon} V(x) \in L^{p}$. Assume that there exist measurable functions $\theta(x, \lambda)$ satisfying the above conditions, such that the operator

$$
(K f)(\lambda)=\int_{0}^{\infty} \theta(x, \lambda)^{2} \exp \left(\frac{i}{\operatorname{Im}\left(\theta \bar{\theta}^{\prime}\right)} \int_{0}^{x} V(t)|\theta(t, \lambda)|^{2} d t\right) f(x) d x
$$

satisfies an $L_{2}\left(\mathbb{R}^{+}, d x\right)-L^{2}(S, d \lambda)$ bound on functions $f$ of compact support. Then the absolutely continuous spectrum of $H_{U}$ supported on the set $S$ is preserved under perturbation by $V$, that is, the set $S$ belongs to an essential support of the absolutely continuous part of the spectral measure of operator $H_{U+V}$. Moreover, for almost every $\lambda \in S$, there exist solutions $\psi_{\lambda}(x), \bar{\psi}_{\lambda}(x)$ of the equation (1) with the asymptotic behavior (2).

Remarks. 1. The assumption of the boundedness of all solutions at almost all energies corresponding to the essential support of absolutely continuous spectrum is rather natural. Almost all known examples of one-dimensional Schrödinger operators with absolutely continuous spectrum satisfy this assumption. Only recently there appeared rigorous counterexamples to the conjecture that this is true in general (see [19]), but the corresponding potentials are of a rather special form and in particular are not bounded from below.

2. All three main theorems that we prove have natural analogues for the whole axis problems. We will not focus on this aspect; all proofs may be generalized to the whole axis case in a straightforward manner following [14].

3 . We have not been able to treat potentials that are assumed merely to belong to $L^{p}$ for some $1<p \leq 2$.

The main new technique we developed in this paper involves norm bounds and almost everywhere convergence results for a class of multilinear integral operators, which may be of interest in its own right. The plan of the paper is as follows. In Section 2 we discuss the basic scheme of asymptotic integration. In Section 3 we formulate the first key results on estimates for maximal functions of certain integral operators. In Section 4 we establish norm estimates for multilinear integral operators, and in Section 5 prove corresponding a.e. convergence results. Section 6 contains the conclusion of the proof of all the main results. In the Appendix we discuss generalization of our results to the case of potentials which may have strong local singularities.

Independently, results similar to Theorem 1.1 were obtained by Remling [25] by a very different method, based in part on the ideas from [14] and [21]. Some of the results proved here (along with some of the results of [25]) were announced in [8]. That announcement also contains a list of open problems which we find most interesting.

\section{AsYmptotic IntEgRATion AND BOUNDED EIGENFUnCtions}

To prove the stability of the absolutely continuous spectrum of the operator $H_{U}$, we will use the following Lemma: 
Lemma 2.1. Let the potential $W(x)$ be locally integrable and satisfy

$$
\sup _{x} \int_{|x-t| \leq 1}\left|W_{-}(t)\right| d t<\infty
$$

( $W_{-}$denotes the negative part of the potential $W$ ). Suppose that for every energy $\lambda$ from a set $E$ of positive Lebesgue measure, all solutions of the equation

$$
-\frac{d^{2}}{d x^{2}} y(x)+W(x) y(x)=\lambda y(x)
$$

are bounded. Then the set $E$ is contained in an essential support of the absolutely continuous part of the spectral measure of the operator $H_{W}$.

The proof of this Lemma may be found in [29] (see also [27]). We note that in different formulations, the fact that bounded solutions imply absolutely continuous spectrum has been known for a long time (see, e.g., [6]). Lemma 2.1 is the most convenient statement for our purpose.

The general plan of our proof is similar to [14] and may be described as follows. By assumption, we know that for every $\lambda \in S$, all solutions of the generalized eigenfunction equation (3) for the unperturbed operator are bounded. Examples with imbedded eigenvalues $[22,26,17]$ show that if $V(x)$ is not short-range, we cannot hope in general that for every $\lambda \in S$ we still have only bounded solutions for a perturbed equation; there may exist rather a rich, dense in $S$ set for which we will have decaying $\left(L^{2}\right)$ and therefore also growing solutions. Our goal will be to show that nevertheless for a.e. $\lambda \in S$, we still have only bounded solutions. This will ensure that the absolutely continuous spectrum is preserved, although embedded singular spectrum may occur.

Thus, our goal is to study the solutions of the equation

$$
-y^{\prime \prime}+(U(x)+V(x)) y=\lambda y .
$$

We rewrite this equation as a system

$$
y_{1}^{\prime}=\left(\begin{array}{cc}
0 & 1 \\
U+V-\lambda & 0
\end{array}\right) y_{1},
$$

where $y_{1}$ is now a vector $\left(\begin{array}{c}y \\ y^{\prime}\end{array}\right)$. Let us apply a variation of parameters transformation with solutions of the unperturbed equation

$$
y_{1}=\left(\begin{array}{cc}
\theta(x, \lambda) & \bar{\theta}(x, \lambda) \\
\theta^{\prime}(x, \lambda) & \bar{\theta}^{\prime}(x, \lambda)
\end{array}\right) y_{2}
$$

to bring the equation to a more symmetric form

$$
y_{2}^{\prime}=\frac{i}{2 \operatorname{Im}\left(\theta \bar{\theta}^{\prime}\right)}\left(\begin{array}{cc}
V(x)|\theta(x, \lambda)|^{2} & V(x) \bar{\theta}(x, \lambda)^{2} \\
-V(x) \theta(x, \lambda)^{2} & -V(x)|\theta(x, \lambda)|^{2}
\end{array}\right) y_{2} .
$$

Notice that $2 i \operatorname{Im}\left(\theta, \overline{\theta^{\prime}}\right)$ is a Wronskian of two solutions $\theta$ and $\bar{\theta}$ and hence is independent of $x$. Let us introduce the notation

$$
p(x, \lambda)=\frac{1}{2 \operatorname{Im}\left(\theta \overline{\theta^{\prime}}\right)} \int_{0}^{x} V(y)|\theta(y, \lambda)|^{2} d y .
$$


It will be convenient to apply to (5) an additional transformation

$$
y_{2}=\left(\begin{array}{cc}
\exp (i p(x, \lambda)) & 0 \\
0 & \exp (-i p(x, \lambda))
\end{array}\right) y_{3} .
$$

We arrive at the following equation for $y_{3}$ :

$(6)$

$$
y_{3}^{\prime}=\frac{i}{2 \operatorname{Im}\left(\theta \bar{\theta}^{\prime}\right)}\left(\begin{array}{cc}
0 & V(x) \bar{\theta}(x, \lambda)^{2} \exp (-2 i p(x, \lambda)) \\
-V(x) \theta(x, \lambda)^{2} \exp (2 i p(x, \lambda)) & 0
\end{array}\right) y_{3} .
$$

We follow the idea of " $(I+Q)$ " asymptotic integration originating in Harris-Lutz [10]: to find some invertible transformation of equation (6) which would make the off-diagonal terms absolutely integrable and then apply Levinson's theorem (see, e.g. [9]) to find the asymptotic behavior of solutions of the resulting equation. If we succeed, we can go back and find the asymptotic behavior of the solutions of the original equation.

Let

$$
y_{3}=\left(1-|q|^{2}\right)^{-\frac{1}{2}}(I+Q) y_{4},
$$

where $I$ is an identity matrix,

$$
Q=\left(\begin{array}{cc}
0 & q \\
\bar{q} & 0
\end{array}\right)
$$

and $q(x, \lambda)$ is some function to be defined. A computation gives for $y_{4}$

$$
\begin{aligned}
& y_{4}^{\prime}=\left(1-|q|^{2}\right)^{-1}\left[\frac{i}{2 \operatorname{Im}\left(\theta \bar{\theta}^{\prime}\right)}\right. \\
& V \operatorname{Re}\left(\theta^{2} q \exp (2 i p)\right) \\
& \left(\begin{array}{cc}
V \bar{\theta}^{2} \exp (-2 i p)+V \theta^{2} q^{2} \exp (2 i p) \\
-V \theta^{2} \exp (2 i p)-V \bar{\theta}^{2} \bar{q}^{2} \exp (-2 i p) & -V \operatorname{Re}\left(\theta^{2} q \exp (2 i p)\right)
\end{array}\right) y_{4} \\
& \left.+\left(\begin{array}{cc}
\frac{1}{2}\left(q \bar{q}^{\prime}-q^{\prime} \bar{q}\right) & -q^{\prime} \\
-\bar{q}^{\prime} & \frac{1}{2}\left(q^{\prime} \bar{q}-q \bar{q}^{\prime}\right)
\end{array}\right) y_{4}\right]
\end{aligned}
$$

We summarize the main result of this section in

Theorem 2.2. Suppose that for some given $\lambda$ there exists a function $q(x, \lambda) \in C^{1}$, such that $q(x, \lambda) \rightarrow 0$ as $x \rightarrow+\infty$, and such that

$$
\begin{aligned}
q^{\prime}(x, \lambda)- & \frac{i}{2 \operatorname{Im}\left(\theta \bar{\theta}^{\prime}\right)} V(x) \bar{\theta}(x, \lambda)^{2} \exp (-2 i p(x, \lambda)) \\
& -\frac{i}{2 \operatorname{Im}\left(\theta \bar{\theta}^{\prime}\right)} V(x) \theta(x, \lambda)^{2} \exp (2 i p(x, \lambda)) q^{2}(x, \lambda) \in L^{1}((0, \infty), d x) .
\end{aligned}
$$

Then all solutions of the generalized eigenfunction equation (1) for this parameter $\lambda$ are bounded. Moreover, there are two solutions $\psi(x, \lambda), \bar{\psi}(x, \lambda)$ with the asymptotic behavior

$$
\begin{aligned}
\psi(x, \lambda)= & \theta(x, \lambda) \exp (i p(x, \lambda)) \\
& \cdot \exp \left(\frac{i}{2 \operatorname{Im}\left(\theta \bar{\theta}^{\prime}\right)} \int_{0}^{x}\left(1-|q|^{2}\right)^{-1} V \operatorname{Re}\left(\theta^{2} q \exp (2 i p)\right) d t\right)(1+o(1))
\end{aligned}
$$

as $x \rightarrow \infty$. 
Remark. In all our applications, the integrand in the cumbersome final factor in the product giving the asymptotic behavior will turn out to be integrable, so this factor will itself be $1+o(1)$.

Proof. The proof follows immediately from Levinson's theorem (see, e.g. [9]) and equation (8). We may consider this system of equations only for $x$ large enough, so that $|q(t, \lambda)|<1$ for all $t>x$ and the transformation (7) is non-singular. By the assumption of the theorem, the off-diagonal terms are absolutely integrable. The diagonal terms are purely imaginary and hence Levinson's theorem is applicable. Asymptotic behavior of the solutions (10) follows directly from the explicit solution of the equation (8) with diagonal terms omitted and transformations we applied to the original system of equations.

To complete the proof of Theorem 1.3, we need to construct the function $q(x, \lambda)$ satisfying (9) and conditions given in Theorem 2.2 for almost every $\lambda \in S$. Solving the equation

$$
\begin{aligned}
q^{\prime}(x, \lambda)- & \frac{i}{2 \operatorname{Im}\left(\theta \bar{\theta}^{\prime}\right)} V(x) \bar{\theta}(x, \lambda)^{2} \exp (-2 i p(x, \lambda)) \\
& \quad-\frac{i}{2 \operatorname{Im}\left(\theta \bar{\theta}^{\prime}\right)} V(x) \theta(x, \lambda)^{2} \exp (2 i p(x, \lambda)) q^{2}(x, \lambda)=0
\end{aligned}
$$

by iteration leads us to expressions involving certain multilinear integral operators. We need to show that these expressions converge for a.e. $\lambda \in S$ in order to ensure $q(x, \lambda) \stackrel{x \rightarrow \infty}{\longrightarrow} 0$ for a.e. $\lambda$, and to make sure that (9) is satisfied after some finite number of iterations. The first approximation to the solution would be

$$
q^{(0)}(x, \lambda)=-\frac{i}{2 \operatorname{Im}\left(\theta \bar{\theta}^{\prime}\right)} \int_{x}^{\infty} V(t) \bar{\theta}(t, \lambda)^{2} \exp (-2 i p(t, \lambda)) d t .
$$

Again, we have to justify this formula by proving that the conditional integral is well-defined for almost every $\lambda$. This is relatively simple and has been already done in [14], but the multilinear expressions arising in higher-order approximations require further analysis. In the next section, we formulate the main result from [14] that we will use and make a few comments.

\section{Almost EVERYWhere CONVERGEnCE FOR INTEGRAL OPERATORS}

Let an operator $K$ be defined on all measurable bounded functions $f$ of compact support by

$$
(K f)(\lambda)=\int_{0}^{\infty} k(\lambda, x) f(x) d x,
$$

where $k(\lambda, x)$ is a measurable and bounded function on $I \times \mathbb{R}^{+}$. To study the a.e. convergence of the integral defining $K f(\lambda)$ on functions from $L^{p}$, we study the corresponding maximal function. Denote by $M_{K} f(\lambda)$ the maximal function

$$
M_{K} f(\lambda)=\sup _{N}\left|\int_{0}^{N} k(\lambda, x) f(x) d x\right| .
$$

The following is a mild generalization of a result proved in [14]. 
Theorem 3.1. Let $p, q$ be exponents satisfying $1 \leq p<q \leq \infty$. Suppose that $K$ is a bounded linear operator from $L^{p}(\mathbb{R})$ to $L^{q}(\mathbb{R})$. Then the maximal function $M_{K}$ also maps $L^{p}$ to $L^{q}$ boundedly, that is,

$$
\left\|M_{K} f\right\|_{q} \leq C\|f\|_{p} \text { for every } f \in L^{p} .
$$

As a consequence, the integral

$$
\int_{0}^{N} k(\lambda, x) f(x) d x
$$

converges as $N \rightarrow \infty$ for almost every value of $\lambda$, for every $f \in L^{p}$.

The next theorem is essentially more general. Denote by $\|K\|_{p, q}$ the norm of $K$, as an operator from $L^{p}$ to $L^{q}$. By $\chi_{E}(x)$ we denote a characteristic function which is equal to one when $x \in E$ and is zero otherwise.

Theorem 3.2. Let $(X, \mu),(Y, \nu)$ be measure spaces. Suppose that $1 \leq p<q \leq \infty$. Then for any bounded linear operator $K$ from $L^{p}(X)$ to $L^{q}(Y)$, and for any sequence of measurable sets $\left\{E_{n} \subset X: n \in Z\right\}$ such that $E_{n} \subset E_{n+1}$ for every $n$, the maximal function

$$
M_{K} f(y)=\sup _{n}\left|K\left(f \cdot \chi_{E_{n}}\right)(y)\right|
$$

is bounded from $L^{p}(X)$ to $L^{q}(Y)$. Moreover

$$
\left\|M_{K}\right\|_{p, q} \leq A\|K\|_{p, q}
$$

where $A<\infty$ depends only on $p, q$.

This simple result is proved in [7]; see [15] for other related results. ${ }^{2}$ To deduce Theorem 3.1, let $R$ be a large parameter, and take $E_{n}=[0, n / R]$. Letting $R \rightarrow \infty$ leads to the first conclusion of the theorem. With boundedness of the maximal function in hand, the almost everywhere convergence conclusion follows, since it holds for the dense class consisting of all compactly supported functions.

\section{Norm estimates FOR MUltilinear transforms}

In this section, we study norm estimates for certain multilinear transforms. The results of this section and the next will enable us to fulfill the plan sketched at the end of Section 2 and to find the function $q(x, \lambda)$ with the needed properties for a.e. $\lambda$ by iteration of (11).

Suppose that the functions $k_{i}(\lambda, x), i=1, \ldots, n$, are defined on $I \times \mathbb{R}^{+}$, where $I$ is some measurable set in $\mathbb{R}$. We assume that the operators

$$
\left(K_{i} f\right)(\lambda)=\int_{\mathbb{R}^{+}} k_{i}(\lambda, x) f(x) d x
$$

satisfy the bounds

$$
\left\|K_{i} f\right\|_{L^{q}(I, d \lambda)} \leq C_{i}\|f\|_{L^{p}\left(\mathbb{R}^{+}, d x\right)}
$$

on functions of compact support for some $2>p \geq 1$ and $q>p$.

Let $n \geq 2$. Let $A$ be any set of ordered pairs $\alpha=\left(i_{\alpha}, i_{\alpha}^{\prime}\right)$, with $1 \leq i_{\alpha}, i_{\alpha}^{\prime} \leq n$. Let $|A|$ denote the cardinality of $A$.

\footnotetext{
${ }^{2}$ The mechanism underlying the proof of Theorem 3.2 is presented in the proofs of Theorem 4.1 and Lemma A.3 below; Theorems 3.1 and 3.2 may be deduced via simple modification of those proofs.
} 
Consider the multilinear operator $T_{n}$ given by

$$
T_{n}\left(f_{1}, \ldots, f_{n}\right)(\lambda)=\int_{\mathbb{R}^{n}} \prod_{j=1}^{n} f_{j}\left(x_{j}\right) k_{j}\left(x_{j}, \lambda\right) \prod_{\alpha \in A} \chi_{\mathbb{R}^{+}}\left(x_{i_{\alpha}}-x_{i_{\alpha}^{\prime}}\right) d x,
$$

$x=\left(x_{1}, \ldots, x_{n}\right)$. Notice that if there were no "diagonal" characteristic functions, the expression (16) would decompose into a product of one-dimensional integrals, and the analysis would become trivial.

Remark. We do not rule out the possibility that some of the characteristic functions in (16) are mutually contradictory in the sense that both $\chi_{\mathbb{R}^{+}}\left(x_{i}-x_{j}\right)$ and $\chi_{\mathbb{R}^{+}}\left(x_{j}-x_{i}\right)$ appear; in such a case the integrand vanishes identically.

Our goal in this section is to prove the following property:

Theorem 4.1. Suppose that the multilinear operator $T_{n}$ is given by (16) with kernels $k_{j}\left(\lambda, x_{j}\right)$ satisfying (15). Then for any functions $f_{i} \in L^{p}\left(\mathbb{R}^{+}, d x\right), i=1, \ldots, n$, such that the integral (16) converges absolutely for a.e. $\lambda$, we have

$$
\left\|T_{n}\left(f_{1}, \ldots, f_{n}\right)\right\|_{s_{n}} \leq C_{n} \prod_{i=1}^{n}\left\|f_{i}\right\|_{p},
$$

where $s_{n}^{-1}=n q^{-1}$. The constant $C_{n}$ depends only on $n$ and constants in the norm bounds (15) for the operators $K_{i}$.

Remarks. The conclusion of Theorem 4.1 holds in particular when $s_{n}<1$. Our proof can be made to yield a more general inequality, in which $f_{j} \in L^{p_{j}}$, the exponents $p_{j}$ vary freely in a certain range, and $s_{n}^{-1}=\sum_{j} q_{j}^{-1}$ where $q_{j}^{-1}=1-p_{j}^{-1}$; the case where all exponents $p_{j}$ are equal suffices for our applications, and we restrict attention to it in order to simplify computations slightly.

By assumption, the value of $T_{n}\left(f_{1}, \ldots, f_{n}\right)(\lambda)=g(\lambda)$ is well-defined for a.e. $\lambda$ by the absolutely convergent integral. Our strategy will be to divide the domain of integration into disjoint pieces and to represent the function $g$ as a sum of terms coming from integration over these disjoint pieces; formally:

$$
g(\lambda)=\sum_{i=1}^{\infty} g_{i}(\lambda)
$$

Because of the absolute convergence, we have that the sum $\sum_{i=1}^{n} g_{i}(\lambda)$ converges to $g(\lambda)$ for a.e. $\lambda$ as $n \rightarrow \infty$. We show, choosing the functions $g_{i}$ in a convenient way, that the sum also converges absolutely in the appropriate space $L^{s_{n}}$, thus proving Theorem 4.1.

The proof of Theorem 4.1 will exploit a special representation of the function

$$
f\left(x_{1}\right) f\left(x_{2}\right) \chi_{\mathbb{R}^{+}}\left(x_{2}-x_{1}\right)
$$

as a sum of products of two functions depending only on $x_{1}$ and $x_{2}$ respectively. Let us first introduce a decomposition of $\mathbb{R}^{+}$associated with the function $f$. Normalize $f$ so that $\|f\|_{p}^{p}=1$. Let $E(1,1)$ and $E(1,2)$ be disjoint intervals such that

$$
\left\|f(x) \chi_{E(1,1)}\right\|_{p}^{p}=\left\|f(x) \chi_{E(1,2)}\right\|_{p}^{p}=2^{-1},
$$

$E(1,1) \cup E(1,2)=\mathbb{R}^{+}$and $E(1,1)$ lies entirely to the left of $E(1,2)$ (i.e. for any $x \in E(1,1), y \in E(1,2)$ we have $x<y)$. We note that $E(1,2)$ is half-infinite and assume $E(1,1)$ contains its right end for the above decomposition to hold. We also 
remark that the decomposition is not necessarily unique (if $f$ vanishes on some interval, then it will be non-unique), and we just choose one such decomposition. In the future we will omit such inessential details. We continue to decompose each of the intervals $E(1, i)$ in a similar manner, obtaining on the $m^{\text {th }}$ step $2^{m}$ intervals $\{E(m, l)\}_{l=1}^{2^{m}}$, such that $\bigcup_{l=1}^{2^{m}} E(m, l)=\mathbb{R}^{+},\|f(x) \chi(E(m, l))\|_{p}^{p}=2^{-m}$ for $j=1, \ldots, 2^{m}$, the intervals are disjoint and $E(m, l)$ lies entirely to the left of $E(m, i)$ if $l<i$. In the notation $E(m, l)$, we refer to $m$ as the "generation" of this interval and to $l$ as the "index". Of importance, in particular, will be the following evident property of intervals $\left\{E(m, l): 1 \leq m \leq \infty, 1 \leq l \leq 2^{m}\right\}$ : any two intervals are either disjoint or one is contained in another.

We proceed to decompose the "diagonal" characteristic functions in a convenient way.

Lemma 4.2. The following identity holds:

$$
\chi_{\mathbb{R}^{+}}\left(x_{2}-x_{1}\right) f\left(x_{1}\right) f\left(x_{2}\right)=\left(\sum_{m=1}^{\infty} \sum_{\substack{l=1 \\ l \text { odd }}}^{2^{m}} \chi_{E(m, l)}\left(x_{1}\right) \chi_{E(m, l+1)}\left(x_{2}\right)\right) f\left(x_{1}\right) f\left(x_{2}\right) .
$$

Proof. Figure 1 illustrates the decomposition that we perform. Let us denote by $H_{12}$ the set

$$
H_{12}=\left\{x \in \mathbb{R}^{2}, x=\left(x_{1}, x_{2}\right) \mid x_{1}<x_{2}\right\}
$$

and by $\operatorname{supp} f$ the set of the points $x$ such that for every interval $I$, such that $x \in I$, $|f(x)|$ is positive on the set of positive Lebesgue measure in $I$. The claim will follow if we show that

$$
H_{12} \cap\left(\operatorname{supp}_{x_{1}} f \times \operatorname{supp}_{x_{2}} f\right)=\bigcup_{m=1}^{\infty} \bigcup_{\substack{l=1 \\ l \text { odd }}}^{2^{m}}(E(m, l) \times E(m, l+1))
$$

and the sets under the union on the right-hand side are disjoint. The latter fact is easy to see: if $E(m, l) \subset E(s, i), l$ odd, $s \neq m$, then necessarily $m>s$ and $E(m, l+1)$ also belongs to $E(s, i)$, not $E(s, i+1)$. On the other hand, we show

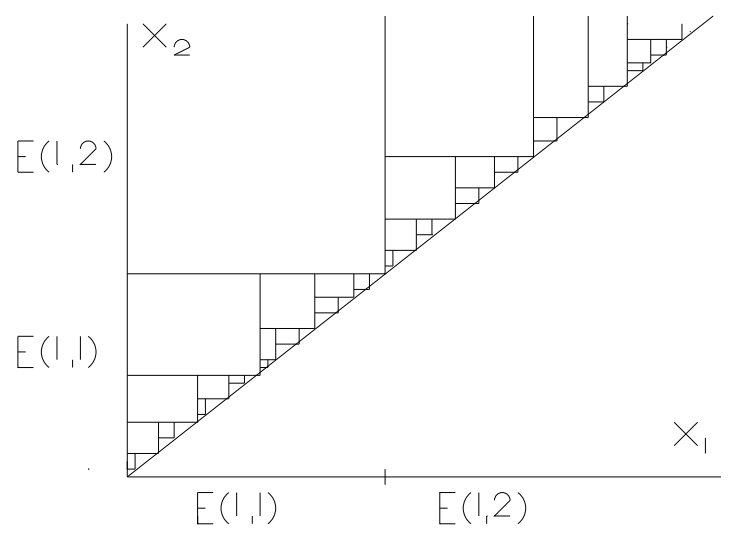

FiguRE 1. Decomposition of $\chi_{\mathbb{R}^{+}}\left(x_{2}-x_{1}\right)$ 
that for every $y_{2}, y_{1} \in \operatorname{supp} f, y_{1}<y_{2}$, there exist two sets $E(m, l), E(m, l+1)$, with $l$ odd, such that $y_{1} \in E(m, l)$ and $y_{2} \in E(m, l+1)$. Let $\left\|f \chi_{\left(y_{1}, y_{2}\right)}\right\|_{p}^{p}=a>0$. Here we assume that $f$ is normalized and use the condition that $y_{1}, y_{2}$ lie in $\operatorname{supp} f$ to infer that $a>0$. Choose $s$ so that $2^{-s} \geq a \geq 2^{-s-1}$. If $y_{1}, y_{2}$ lie in one set of generation $s, E(s, l)$, then necessarily $y_{1} \in E(s+1,2 l-1)$ and $y_{2} \in E(s+1,2 l)$. If $y_{1}$ and $y_{2}$ lie in different sets of generation $m, E(s, l)$ and $E(s, l+1)$, then either $l$ is odd or $y_{1} \in E\left(s-r, l / 2^{r}\right)$ and $y_{2} \in E\left(s-r, l / 2^{r}+1\right)$, where $r$ is such that $l / 2^{r}$ is odd.

Remark. In particular, if $\operatorname{supp} f=\mathbb{R}^{+}$, we get a representation of the diagonal characteristic function $\chi_{\mathbb{R}^{+}}\left(x_{2}-x_{1}\right)$ as a sum of products of characteristic functions of some intervals in the $x_{1}$ and $x_{2}$ variables.

We now begin the proof of Theorem 4.1.

Proof. Since $T_{n}$ is multilinear, we may assume without loss of generality throughout the proof that $\left\|f_{i}\right\|_{p}^{p}=\frac{1}{n}$ for all $i=1, \ldots, n$. Let

$$
f(x)=\left(\sum_{i=1}^{n}\left|f_{i}(x)\right|^{p}\right)^{\frac{1}{p}} .
$$

Consider the family of the intervals $\{E(m, l)\}$ associated with the function $f$. An important property of this family is that

$$
\left\|f_{i}(x) \chi_{E(m, l)}\right\|_{p}^{p} \leq 2^{-m}
$$

for all $i, l, m$. Write

$$
A=\left\{\alpha_{1}, \ldots, \alpha_{|A|}\right\} .
$$

We begin by substituting the result of Lemma 4.2 into formula (16):

$$
\begin{aligned}
T_{n}(\bar{f})=\sum_{m_{1}=1}^{\infty} \cdots \sum_{m_{|A|}=1}^{\infty} \sum_{l_{1}}^{\prime} \cdots \sum_{l_{|A|}}^{\prime} \int_{\mathbb{R}^{n}} d & \prod_{j=1}^{n} k_{j}\left(\lambda, x_{j}\right) f_{j}\left(x_{j}\right) \\
& \cdot \prod_{t=1}^{|A|} \chi_{E\left(m_{t}, l_{t}\right)}\left(x_{i_{t}}\right) \chi_{E\left(m_{t}, l_{t}+1\right)}\left(x_{i_{t}^{\prime}}\right),
\end{aligned}
$$

where $\sum_{l_{t}}^{\prime}$ means the sum over all odd integers $l_{t} \in\left[1,2^{m_{t}}\right]$, and $i_{t}=i_{\alpha_{t}}, i_{t}^{\prime}=i_{\alpha_{t}}^{\prime}$. Thus

$$
\left|T_{n}(\bar{f})(\lambda)\right| \leq \sum_{m_{1}=1}^{\infty} \cdots \sum_{m_{|A|}=1}^{\infty} F^{\bar{m}}(\bar{f})(\lambda),
$$

where $\bar{m}=\left(m_{1}, \ldots, m_{|A|}\right)$ and

$$
F^{\bar{m}}(\bar{f})(\lambda)=\sum_{l_{1}}^{\prime} \cdots \sum_{l_{|A|}}^{\prime} \int_{\mathbb{R}^{n}} d x \prod_{j=1}^{n}\left|K_{j}\left(f_{j} \chi_{G(j, \bar{l})}\right)(\lambda)\right|,
$$


where $\bar{l}=\left(l_{1}, \ldots, l_{|A|}\right)$ (all variables $l_{t}$ take only odd values), the set $G(j, \bar{l})$ depends on $\bar{m}$ (and on $A$ ), and

$$
G(j, \bar{l})=\left[\bigcap_{t: j=i_{t}} E\left(m_{t}, l_{t}\right)\right] \cap\left[\bigcap_{t: j=i_{t}^{\prime}} E\left(m_{t}, l_{t}+1\right)\right] .
$$

We aim to prove that

$$
\left\|F^{\bar{m}}(\bar{g})\right\|_{s_{n}} \leq C_{n} 2^{-\gamma_{n}|\bar{m}|} \prod_{j=1}^{n}\left\|g_{j}\right\|_{p}^{\left(1-\beta_{n}\right)}
$$

for any $g_{1}, \ldots, g_{n}$ which satisfy (18) (that is, $\left\|g_{i} \chi_{E(m, l)}\right\|_{p}^{p} \leq 2^{-m}$ for all $i, m, l$ ). Here $\gamma_{n}$ is some positive constant which depends only on $n, C_{n}$ depends on $n$ and constants in norm bounds (15) for the operators $K_{j}$, and $\beta_{n}$ satisfies

$$
1 \geq 1-\beta_{n}>\max \left(\frac{p}{q}, \frac{p}{2}\right) .
$$

Invoking the decomposition (20) and summing over $\bar{m}$, Theorem 4.1 follows directly from $(22)$.

We may proceed to prove (22) by induction on $|A|$. The case $|A|=0$ is immediate from the hypothesis on $K_{j}$ by Hölder's inequality (in this case $\bar{m}$ is a 0 -tuple). It will be convenient to consider the graph $\Upsilon$ with vertices $\{1, \ldots, n\}$ and edges $\left(i_{t}, i_{t}^{\prime}\right)$ joining $i_{t}$ to $i_{t}^{\prime}$ for any $t$ (and no other edges). To each edge we associate the generation $m_{t}$ which corresponds to a generation in the decomposition (17) of $\chi_{\mathbb{R}^{+}}\left(x_{i_{t}}-x_{i_{t}^{\prime}}\right)$ that we fixed in the sum (21). It suffices to treat the case where $\Upsilon$ is connected; the general case then follows by Hölder's inequality.

Fix $\bar{m}$. Relabel the indices so that $m_{1} \leq \ldots \leq m_{|A|}$. For simplicity of notation, we also relabel pairs $\left(i_{t}, i_{t}^{\prime}\right)$ so that $m_{t}$ still denotes the generation in the decomposition of $\chi_{\mathbb{R}^{+}}\left(x_{i_{t}}-x_{i_{t}^{\prime}}\right)$. Let $N$ be the largest index for which $m_{N}=m_{1}$.

For many values of $\bar{l}$, the set $G(j, \bar{l})$ is empty for some $j$. Such terms contribute zero to the sum (21); this observation underlies the estimate $(22)$ for $F^{\bar{m}}(\bar{f})$. To take this into account, drop from the sum (21) all terms for which there exists $j$ such that $G(j, \bar{l})=\emptyset$; such terms contribute 0 to $F^{\bar{m}}(\bar{g})$. We say that an index $\bar{l}$ remains if the corresponding term has not been dropped.

We have the following

Lemma 4.3. For any $1 \leq j \leq n$, either

$$
G(j, \bar{l}) \subset E\left(m_{1}, l_{1}\right) \forall \bar{l} \text { remaining }
$$

or

$$
G(j, \bar{l}) \subset E\left(m_{1}, l_{1}+1\right) \forall \bar{l} \text { remaining. }
$$

Let $B_{1}=\{j:$ (23) holds $\}, B_{2}=\{j:(24)$ holds $\}$. For any $2 \leq t \leq N$ (that is, if $\left.m_{t}=m_{1}\right)$,

$$
l_{t}=l_{1} \forall \bar{l} \text { remaining } .
$$

Finally for each $t>N$ (so $\left.m_{t}>m_{1}\right)$, either $i_{t}, i_{t}^{\prime}$ are both in $B_{1}$ for all remaining $l$ or they are both in $B_{2}$ for all remaining $l$. We say that $t \in A_{1}, t \in A_{2}$ respectively.

Proof. For any $m \geq 1, l$ odd, set

$$
\tilde{E}(m, l)=E(m, l) \cup E(m, l+1) .
$$


First we prove that if $\bar{l}$ remains, then

$$
\tilde{E}\left(m_{t}, l_{t}\right) \subset \tilde{E}\left(m_{1}, l_{1}\right)
$$

for all $t$. Notice that both sets in (26) also belong to the family $E(m, l)$ (they are $E\left(m_{t}-1, \frac{l_{t}-1}{2}\right), E\left(m_{1}-1, \frac{l_{1}-1}{2}\right)$ respectively; we may assume $\left.E(0,0)=\mathbb{R}^{+}\right)$. Therefore, to prove (26) it is sufficient to show that the two sets in (26) intersect, since in this case one is contained in another by the martingale-type property.

Recall that $m_{1}$ is the generation which is fixed in decomposition of the characteristic function $\chi_{\mathbb{R}^{+}}\left(x_{i_{1}}-x_{i_{1}^{\prime}}\right)$ in the sum (21) for $F^{\bar{m}}$. Pick any other $m_{t}$ which is fixed in the decomposition of the characteristic function $\chi_{\mathbb{R}^{+}}\left(x_{i_{t}}-x_{i_{t}^{\prime}}\right)$. Since the graph $\Upsilon$ is connected, we can find a path in $\Upsilon$ which connects either $i_{t}$ or $i_{t}^{\prime}$ with either $i_{1}$ or $i_{1}^{\prime}$, and does not contain the edges $\left(i_{1}, i_{1}^{\prime}\right),\left(i_{t}, i_{t}^{\prime}\right)$. Suppose that this path goes from $i_{1}$ to $i_{t}$ and passes successively through the edges with the corresponding generations $m_{t_{1}}, \ldots, m_{t_{r}}$. This path does not depend on $\bar{l}$.

For $G(j, \bar{l})$ to be non-zero for all $j$, we must have

$$
\begin{aligned}
& \tilde{E}\left(m_{1}, l_{1}\right) \cap \tilde{E}\left(m_{t_{1}}, l_{t_{1}}\right) \neq \emptyset, \quad \tilde{E}\left(m_{t}, l_{t}\right) \cap \tilde{E}\left(m_{t_{r}}, l_{t_{r}}\right) \neq \emptyset, \\
& \text { and } \tilde{E}\left(m_{t_{i}}, l_{t_{i}}\right) \cap \tilde{E}\left(m_{t_{i+1}}, l_{t_{i+1}}\right) \neq \emptyset \text { for all } i=1, \ldots, r-1 .
\end{aligned}
$$

Hence by our assumption that $m_{1} \leq m_{t}$ for all $t$ we see that $\tilde{E}\left(m_{t_{1}}, l_{t_{1}}\right) \subset \tilde{E}\left(m_{1}, l_{1}\right)$. But then by $(27)$ also $\tilde{E}\left(m_{1}, l_{1}\right) \cap \tilde{E}\left(m_{t_{2}}, l_{t_{2}}\right) \neq \emptyset$, hence $\tilde{E}\left(m_{t_{2}}, l_{t_{2}}\right) \subset \tilde{E}\left(m_{1}, l_{1}\right)$. We continue in the same way concluding that $\tilde{E}\left(m_{t}, l_{t}\right) \subset \tilde{E}\left(m_{1}, l_{1}\right)$ and hence (26) holds.

The statements (23), (24) and (25) of the lemma now follow immediately from the martingale-type property of the sets $E(m, l)$ and the definition of the set $G(j, \bar{l})$. To obtain (25), note that because $l_{1}$ and $l_{t}$ are odd, when $l_{t}=l_{1}$ the inclusion $E\left(m_{t}, l_{t}\right) \subset \tilde{E}\left(m_{1}, l_{1}\right)$ forces $E\left(m_{t}, l_{t}\right)=E\left(m_{1}, l_{1}\right)$. To prove the final statement, suppose that we know in addition that $m_{t}>m_{1}$. We can find a path in $\Upsilon$ which goes from $i_{t}$ or $i_{t}^{\prime}$ to a vertex adjacent to an edge $\left(i_{s}, i_{s}^{\prime}\right)$ with the corresponding generation $m_{s}$ equal to $m_{1}$ (i.e. with $s \leq N$ ), and contains only edges with the corresponding generations strictly less than $m_{1}$. An argument analogous to the above shows that in this case $\tilde{E}\left(m_{t}, l_{t}\right)$ is contained either in $E\left(m_{1}, l_{s}\right)$ or in $E\left(m_{1}, l_{s}+1\right)$ for all remaining $\bar{l}$, depending on whether the vertex to which the path leads coincides with $i_{s}$ or $i_{s}^{\prime}$ respectively. By (25) the lemma is proven.

Remark. It may happen that there exist two (or more) different paths from $i_{t}$ (or $\left.i_{t}^{\prime}\right)$, one of which leads to a vertex $j_{1}$ where $f_{j_{1}}\left(x_{j_{1}}\right)$ is multiplied by $\chi_{E\left(m_{1}, l_{1}\right)}\left(x_{j_{1}}\right)$, while the other leads to a vertex $j_{2}$ where $f_{j_{2}}\left(x_{j_{2}}\right)$ is multiplied by $\chi_{E\left(m_{1}, l_{1}+1\right)}\left(x_{j_{2}}\right)$. (The simplest way for this to happen is for $A$ to include two pairs $(i, j)$ and $(j, i)$.) In this case, $G(j, \bar{l})$ is zero and hence $\bar{l}$ does not remain.

Define

$$
F_{A_{1}, B_{1}}^{\bar{m}^{(1)}, l_{1}}(\bar{f})(\lambda)=\sum_{l_{t}: t \in A_{1}}^{\prime} \prod_{j \in B_{1}}\left|K_{j}\left(f_{j} \chi_{E\left(m_{1}, l_{1}\right)} \chi_{G(j, l)}\right)(\lambda)\right|
$$

and denote by $\sum_{l_{t}: t \in A_{1}}^{\prime}$ the sum over all $l_{t}$ such that $1 \leq l_{t} \leq 2^{m_{t}}, l_{t}$ is odd, $t \in A_{1}$, where $\bar{m}^{(1)}=\left(m_{t}\right)_{t \in A_{1}}$. Note that $F_{A_{1}, B_{1}^{(1)}}^{\bar{m}_{1}}(\bar{f})(\lambda)$ depends only on those $f_{j}$ for which 
$j \in B_{1}$. The factor $F_{A_{2}, B_{2}}^{\bar{m}^{(2)}, l_{1}}(\bar{f})(\lambda)$ is defined similarly, but $\chi_{E\left(m_{1}, l_{1}\right)}$ is replaced by $\chi_{E\left(m_{1}, l_{1}+1\right)}$. By Lemma 4.3

$$
\begin{aligned}
F^{\bar{m}}(\bar{f})(\lambda)= & \left(\sum_{l_{1}} \sum_{l_{N+1}}^{\prime} \cdots \sum_{l_{|A|}}^{\prime} \prod_{j \in B_{1}}\left|K_{j}\left(f_{j} \chi_{E\left(m_{1}, l_{1}\right)} \chi_{G(j, \bar{l})}\right)(\lambda)\right|\right. \\
& \left.\cdot \prod_{j \in B_{2}}\left|K_{j}\left(f_{j} \chi_{E\left(m_{1}, l_{1}+1\right)} \chi_{G(j, \bar{l})}\right)(\lambda)\right|\right) \\
\leq & \sum_{l_{1}}^{\prime} F_{A_{1}, B_{1}}^{\bar{m}^{(1)}, l_{1}}(\bar{f})(\lambda) F_{A_{2}, B_{2}}^{\bar{m}^{(2)}, l_{1}}(\bar{f})(\lambda) .
\end{aligned}
$$

We may rewrite for each $j \in B_{1}$

$$
G(j, l)=E\left(m_{1}, l_{1}\right) \cap G^{1}\left(j,\left(l_{t}\right)_{t \in A_{1}}\right),
$$

where

$$
G^{1}\left(j,\left(l_{t}\right)_{t \in A_{1}}\right)=\left[\bigcap_{t \in A_{1}, j=i_{t}} E\left(m_{t}, l_{t}\right)\right] \cap\left[\bigcap_{t \in A_{1}, j=i_{t}^{\prime}} E\left(m_{t}, l_{t}+1\right)\right] .
$$

Indeed, by Lemma 4.3 all other sets that enter in the definition of $G(j, l)$ belong to $E\left(m_{1}, l_{1}+1\right)$ and hence are absent for the $\bar{l}$ which remain. Thus $F_{A_{1}, B_{1}}^{\bar{m}^{(1)}, l_{1}}(\bar{f})(\lambda)$ and $F_{A_{2}, B_{2}}^{\bar{m}^{(2)}, l_{1}}(\bar{f})(\lambda)$ are expressions of the same form as the original $F^{\bar{m}}$. Since $0<$ $\left|A_{1}\right|<|A|$, both $F_{A_{1}, B_{1}}^{\bar{m}^{(1)}, l_{1}}(\bar{f})(\lambda)$ and $F_{A_{2}, B_{2}}^{\bar{m}^{(2)}, l_{1}}(\bar{f})(\lambda)$ may be estimated by induction on $|A|$. Therefore

$$
\left\|F_{A_{1}, B_{1}}^{\bar{m}^{(1)}, l_{1}}(\bar{f})(\lambda)\right\|_{s_{\left|B_{1}\right|}} \leq C 2^{-\gamma_{\left|B_{1}\right|}\left|m^{(1)}\right|} \prod_{j \in B_{1}}\left\|f_{j} \chi_{E\left(m_{1}, l_{1}\right)}\right\|_{p}^{1-\beta_{\left|B_{1}\right|}}
$$

a similar bound also holds for $F_{A_{2}, B_{2}}^{\bar{m}^{(2)}, l_{1}}(\bar{f})(\lambda)$.

Using (28), we are ready to estimate $\left\|F^{\bar{m}}(\bar{f})\right\|_{s_{n}}$. We distinguish between two cases: $s_{n} \leq 1$ and $s_{n} \geq 1$. Suppose first that $s_{n} \leq 1$. Then

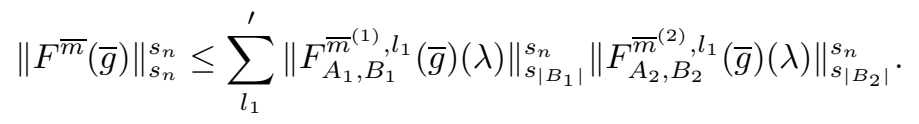

We used the fact that $\left\|\sum h_{i}(x)\right\|_{s}^{s} \leq \sum\left\|h_{i}(x)\right\|_{s}^{s}$ when $s<1$ and Hölder's inequality. Plugging the estimate (28) and a similar bound for $F_{A_{2}, B_{2}}^{\bar{m}^{(2)}, l_{1}}(\bar{g})(\lambda)$ into (29), we find

$$
\begin{aligned}
& \left\|F^{\bar{m}}(\bar{g})\right\|_{s_{n}}^{s_{n}} \leq C_{n} 2^{-\left(\gamma_{\left|B_{1}\right|}\left|m^{(1)}\right|+\gamma_{\left|B_{2}\right|}\left|m^{(2)}\right|\right) s_{n}} \\
& \quad \cdot \sum_{l_{1}}^{\prime}\left(\prod_{j \in B_{1}}\left\|g_{j} \chi_{E\left(m_{1}, l_{1}\right)}\right\|_{p}^{\left(1-\beta_{\left|B_{1}\right|}\right) s_{n}} \prod_{j \in B_{2}}\left\|g_{j} \chi_{E\left(m_{1}, l_{1}+1\right)}\right\|_{p}^{\left(1-\beta_{\left|B_{2}\right|}\right) s_{n}}\right) .
\end{aligned}
$$

Pick $0<a_{1}, a_{2}<1$ such that

$$
a_{1}\left(1-\beta_{\left|B_{1}\right|}\right)=a_{2}\left(1-\beta_{\left|B_{2}\right|}\right)=\frac{p}{q} .
$$


We can find such $a_{1}, a_{2}$ by the induction assumption. The sum in (30) may be estimated by Hölder's inequality in the following way:

$$
\begin{aligned}
\sum_{l_{1}}^{\prime}\left(\prod_{j \in B_{1}}\left\|g_{j} \chi_{E\left(m_{1}, l_{1}\right)}\right\|_{p}^{\left(1-\beta_{\left|B_{1}\right|}\right) s_{n}} \prod_{j \in B_{2}}\left\|g_{j} \chi_{E\left(m_{1}, l_{1}+1\right)}\right\|_{p}^{\left(1-\beta_{\left|B_{2}\right|}\right) s_{n}}\right) \\
\leq \prod_{j \in B_{1}} \max _{l_{1}}\left\|g_{j} \chi_{E\left(m_{1}, l_{1}\right)}\right\|_{p}^{\left(1-a_{1}\right)\left(1-\beta_{\left|B_{1}\right|}\right) s_{n}} \\
\cdot \prod_{j \in B_{2}} \max _{l_{1}}\left\|g_{j} \chi_{E\left(m_{1}, l_{1}+1\right)}\right\|_{p}^{\left(1-a_{2}\right)\left(1-\beta_{\left|B_{2}\right|}\right) s_{n}} \prod_{j=1}^{n}\left\|g_{j}\right\|_{p}^{\frac{p}{q} s_{n}} .
\end{aligned}
$$

Thus

$$
\begin{aligned}
\left\|F^{\bar{m}}(\bar{g})\right\|_{s_{n}} \leq & C_{n} 2^{-\min \left(\gamma_{\left|B_{1}\right|}, \gamma_{\left|B_{2}\right|}\right)\left(\left|m^{(1)}\right|+\left|m^{(2)}\right|\right)} \\
& \cdot \prod_{j=1}^{n} \sup _{l}\left\|g_{j} \chi_{E\left(m_{1}, l\right)}\right\|_{p}^{\min \left(1-\beta_{\left|B_{1}\right|}, 1-\beta_{\left|B_{2}\right|}\right)-\frac{p}{q}} \prod_{j=1}^{n}\left\|g_{j}\right\|_{p}^{\frac{p}{q}} .
\end{aligned}
$$

Obviously $\left\|g_{j}\right\|_{p} \geq \sup _{l}\left\|g_{j} \chi_{E\left(m_{1}, l\right)}\right\|_{p}$, and $\sup _{l}\left\|g_{j} \chi_{E\left(m_{1}, l\right)}\right\|_{p} \leq 2^{-\frac{m_{1}}{p}}$ by our assumption on $g_{j}$. Pick $\beta_{n}$ so that

$$
\min \left(1-\beta_{\left|B_{1}\right|}, 1-\beta_{\left|B_{2}\right|}\right)>1-\beta_{n}>\max \left(\frac{p}{q}, \frac{p}{2}\right),
$$

and

$$
\gamma_{n}=\min \left(\frac{n}{p}\left[\min \left(1-\beta_{\left|B_{1}\right|}, 1-\beta_{\left|B_{2}\right|}\right)-\left(1-\beta_{n}\right)\right], \gamma_{\left|B_{1}\right|}, \gamma_{\left|B_{2}\right|}\right)
$$

Then

$$
\left\|F^{\bar{m}}(\bar{g})\right\|_{s_{n}} \leq C_{n} 2^{-\gamma_{n}|m|} \prod_{j=1}^{n}\left\|g_{j}\right\|_{p}^{1-\beta_{n}} .
$$

There are only finitely many pairs $B_{1}, B_{2}$ such that $\left|B_{1}\right|+\left|B_{2}\right|=n$, and hence the constants $\gamma_{n}, \beta_{n}$ may be chosen to be independent of $B_{1}, B_{2}$.

The case $s_{n} \geq 1$ is similar. Using the triangle inequality and Hölder's inequality, we get

$$
\begin{aligned}
\left\|F^{\bar{m}}(\bar{g})\right\|_{s_{n}} \leq & \sum_{l_{1}}^{\prime}\left\|F_{A_{1}, B_{1}}^{\bar{m}^{(1)}, l_{1}}(\bar{g})(\lambda)\right\|_{s_{\mid B_{1}} \mid}\left\|F_{A_{2}, B_{2}}^{\bar{m}^{(2)}, l_{1}}(\bar{g})(\lambda)\right\|_{s_{\left|B_{2}\right|}} \\
\leq & C_{n} 2^{-\min \left(\gamma_{\left|B_{1}\right|}, \gamma_{\left|B_{2}\right|}\right)\left(\left|m^{(1)}\right|+\left|m^{(2)}\right|\right)} \\
& \cdot \sum_{l_{1}}^{\prime}\left(\prod_{j \in B_{1}}\left\|g_{j} \chi_{E\left(m_{1}, l_{1}\right)}\right\|_{p}^{\left(1-\beta_{\left|B_{1}\right|}\right)} \prod_{j \in B_{2}}\left\|g_{j} \chi_{E\left(m_{1}, l_{1}+1\right)}\right\|_{p}^{\left(1-\beta_{\left|B_{2}\right|}\right)}\right) .
\end{aligned}
$$

Provided that

$$
\frac{1}{p}\left(\left|B_{1}\right|\left(1-\beta_{\left|B_{1}\right|}\right)+\left|B_{2}\right|\left(1-\beta_{\left|B_{2}\right|}\right)\right)>1,
$$

we can apply the argument similar to the case $s_{n}<1$ to prove (31). But (32) holds for all $\left|B_{1}\right|,\left|B_{2}\right| \geq 1$ since by the induction hypothesis $1-\beta_{r}>\frac{p}{2}$ for all $r<n$. This completes the proof of (22), and hence of Theorem 4.1. 


\section{Almost eVEryWhere CONVERGEnCE OF MUltilinear TransForms}

In the proof of the a.e. convergence, an important role will be played by the following operators. Let $D_{1}(\lambda), \ldots, D_{n}(\lambda)$ be measurable functions of $\lambda$ mapping $I$ to $\mathbb{R}^{+} \cup\{\infty\}$. Let us denote by

$$
T_{n}^{D_{1}(\lambda), \ldots, D_{n}(\lambda)}\left(f_{1}, \ldots, f_{n}\right)(\lambda)
$$

an operator obtained from $T_{n}$ by replacing the kernels $k_{i}\left(\lambda, x_{i}\right), i=1, \ldots, n$, with

$$
\tilde{k}_{i}\left(\lambda, x_{i}\right)=k_{i}\left(\lambda, x_{i}\right) \chi_{\mathbb{R}^{+}}\left(D_{i}(\lambda)-x_{i}\right),
$$

$i=2, \ldots, n$. Throughout this section, we will assume that the kernels $k_{i}(\lambda, x)$ are bounded and the integral operators $K_{i}$ corresponding to these kernels satisfy $L^{2}-L^{2}$ estimates. The results we prove here extend directly to more general situations, however the above conditions are exactly the case that we will need in applications and it is convenient to restrict our attention to it. We give the following natural definition:

Definition 5.1. We say that the operator $T_{n}$ converges on functions $f_{1}, \ldots, f_{n}$ for some $\lambda$ if the expressions

$$
T_{n}^{D_{1}, \ldots, D_{n}}\left(f_{1}, \ldots, f_{n}\right)(\lambda)
$$

converge to a finite limit as $\min _{i} D_{i}$ tends to infinity. Namely, there exists a number, which we denote $T_{n}^{\infty, \ldots, \infty}\left(f_{1}, \ldots, f_{n}\right)(\lambda)$ such that for any $\delta>0$ there exists $N_{\delta}$ such that whenever $\min _{i} D_{i} \geq N_{\delta}$, we have

$$
\left|T_{n}^{\infty, \ldots, \infty}\left(f_{1}, \ldots, f_{n}\right)(\lambda)-T_{n}^{D_{1}, \ldots, D_{n}}\left(f_{1}, \ldots, f_{n}\right)(\lambda)\right|<\delta .
$$

Our first result is the following maximal estimate:

Theorem 5.2. Let

$$
M_{T}\left(f_{1}, \ldots, f_{n}\right)(\lambda)=\sup _{D_{1}, \ldots, D_{n}}\left|T_{n}^{D_{1}, \ldots, D_{n}}\left(f_{1}, \ldots, f_{n}\right)(\lambda)\right| .
$$

Then for any $f_{i} \in L^{p}, 1 \leq p<2$, we have

$$
\left\|M_{T}\left(f_{1}, \ldots, f_{n}\right)\right\|_{s_{n}} \leq C_{n} \prod_{i=1}^{n}\left\|f_{i}\right\|_{p},
$$

where $s_{n}^{-1}=n q^{-1}$ and $q^{-1}+p^{-1}=1$.

Proof. The proof uses a well-known device going back to Kolmogorov and Seliverstov. Namely, it is sufficient to show that for any measurable $D_{i}(\lambda), i=1, \ldots, n$, we have

$$
\left\|T_{n}^{D_{1}(\lambda), \ldots, D_{n}(\lambda)}\left(f_{1}, \ldots, f_{n}\right)\right\|_{s_{n}} \leq C_{n} \prod_{i=1}^{n}\left\|f_{i}\right\|_{p}
$$

with the constant $C_{n}$ independent of the functions $D_{i}(\lambda)$. But the expression $T_{n}^{D_{1}(\lambda), \ldots, D_{n}(\lambda)}\left(f_{1}, \ldots, f_{n}\right)(\lambda)$ is obtained from $T_{n}\left(f_{1}, \ldots, f_{n}\right)(\lambda)$ by replacing the kernels $k_{i}(\lambda, x)$ with the kernels

$$
\tilde{k}_{i}(\lambda, x)=k_{i}(\lambda, x) \chi_{\mathbb{R}^{+}}\left(D_{i}(\lambda)-x_{i}\right) .
$$

By Theorem 3.1, these kernels satisfy the estimates (15) for all $p, q$ such that $p<2$ and $q^{-1}=1-p^{-1}$, with constants in the norm bounds that do not depend on $D_{i}(\lambda)$. Therefore Theorem 4.1 is applicable and directly leads to (33). 
As one may expect, the maximal estimate (33) implies a.e. convergence.

Theorem 5.3. The operator $T_{n}\left(f_{1}, \ldots, f_{n}\right)$ converges for almost every $\lambda$ on any functions $f_{i} \in L^{p}, 1 \leq p<2$.

Proof. Suppose, on the contrary, that for some $\epsilon>0$ we have a set $S_{\epsilon}$ of positive Lebesgue measure such that for any $N$ there exist $D_{i j}>N, i=1, \ldots, n, j=1,2$, such that

$$
\left|T_{n}^{D_{11}, \ldots, D_{n 1}}\left(f_{1}, \ldots, f_{n}\right)(\lambda)-T_{n}^{D_{12}, \ldots, D_{n 2}}\left(f_{1}, \ldots, f_{n}\right)(\lambda)\right|>\epsilon
$$

for every $\lambda \in S_{\epsilon}$. Let us denote $f_{i, N}\left(x_{i}\right)=f_{i}\left(x_{i}\right) \chi_{\mathbb{R}^{+}}\left(N-x_{i}\right)$. For any $D_{i}>N$, we have

$$
\begin{aligned}
& \left|T_{n}^{D_{1}, \ldots, D_{n}}\left(f_{1}, \ldots, f_{n}\right)(\lambda)-T_{n}^{D_{1}, \ldots, D_{n}}\left(f_{1, N}, \ldots, f_{n, N}\right)(\lambda)\right| \\
& \quad \leq \sum_{j=1}^{n}\left|T_{n}^{D_{1}, \ldots, D_{n}}\left(g_{1 j}, \ldots, g_{n j}\right)(\lambda)\right|
\end{aligned}
$$

where $g_{i j}=f_{i, N}$ if $i<j, g_{i j}=f_{i}$ if $i>j$, and $g_{i j}=f_{i}-f_{i, N}$ if $i=j$. In other words, we expand the difference into a telescopic sum. To estimate each term in the sum, we can apply Theorem 5.2. We get

$$
\begin{aligned}
\sup _{D_{1}, \ldots, D_{n}}\left\|T_{n}^{D_{1}, \ldots, D_{n}}\left(f_{1}, \ldots, f_{n}\right)(\lambda)-T_{n}^{D_{1}, \ldots, D_{n}}\left(f_{1, N}, \ldots, f_{n, N}\right)(\lambda)\right\|_{s_{n}}^{s_{n}} \\
\leq \sum_{j=1}^{n}\left\|T_{n}^{D_{1}, \ldots, D_{n}}\left(g_{1 j}, \ldots, g_{n j}\right)(\lambda)\right\|_{s_{n}}^{s_{n}} \\
\leq C_{n} \sum_{j=1}^{n}\left(\prod_{i=1}^{n}\left\|g_{i j}\right\|_{p}\right)^{s_{n}} .
\end{aligned}
$$

Clearly, the right-hand side goes to zero as $N \rightarrow \infty$, since every product contains the norm of $f_{i}-f_{i, N}$ for some $i$. On the other hand, the left-hand side is by assumption bounded from below by $\left(\frac{\epsilon}{2}\right)^{s_{n}}\left|S_{\epsilon}\right|$ for every $N$. This gives a contradiction.

A straightforward adjustment of the above argument allows us to pass for almost every $\lambda$ to the infinite limit in any order (e.g. first in $D_{n}$, then in $D_{n-1}$, and so on).

In order to prove a final Lemma that we will need in the iteration process, we need to consider a smaller class of multilinear operators than we did before. These are exactly the operators that appear in the process of solving equation (11) by iteration.

Definition 5.4. We say that the multilinear transform $T_{n}$ belongs to the class $M_{n}$ if

$$
T_{n}\left(f_{1}, \ldots, f_{n}\right)(\lambda)=\int_{0}^{\infty} \ldots \int_{0}^{\infty} d x_{1} \ldots d x_{n} \prod_{j=1}^{n} k_{j}\left(\lambda, x_{j}\right) f_{j}\left(x_{j}\right) \prod_{j=2}^{n} \chi_{\mathbb{R}^{+}}\left(x_{j}-x_{\sigma(j)}\right),
$$

where the kernels $k_{j}(\lambda, x)$ satisfy (15), and the function $\sigma$ takes values in $1, \ldots, n$ and satisfies $\sigma(j)<j$ for every $j$.

Recall the notation $f_{i, N}(x)=f_{i}(x) \chi_{\mathbb{R}^{+}}(N-x)$. 
Lemma 5.5. Suppose that for some $\epsilon>0$ and $1 \leq p<2$, for each index $1 \leq i \leq n$, a function $f_{i}$ satisfies $(1+x)^{\epsilon} f_{i}(x) \in L^{p}$. Then for every $\delta<\epsilon$, for almost every $\lambda \in R$, there exists $C(\lambda, n)<\infty$ such that for every nonnegative $N \in R$,

$$
\left|T_{n}\left(f_{1}-f_{1, N}, f_{2}, \ldots, f_{n}\right)(\lambda)\right| \leq C(\lambda, n)(1+N)^{-n \delta} .
$$

Proof. The structure of operators of class $M_{n}$ is such that the integral defining $T_{n}$ extends only over those $x$ satisfying $x_{j} \geq x_{1}$ for every $j$, because of the requirement that $\sigma(j)<j$ for every $j>1$. Since $f_{1}-f_{1, N}$ is supported where $x>N$, we therefore have for every $N, \lambda$

$$
T_{n}\left(f_{1}-f_{1, N}, f_{2}, \ldots, f_{n}\right)(\lambda)=T_{n}\left(f_{1}-f_{1, N}, f_{2}-f_{2, N}, \ldots, f_{n}-f_{n, N}\right)(\lambda) .
$$

Fix $f_{1}, \ldots, f_{n}$. Since $\left\|f_{j}-f_{j, N}\right\|_{p} \leq C \cdot(1+N)^{-\epsilon}$ by hypothesis, Theorem 5.2 now yields

$$
2^{n r \epsilon}\left\|\sup _{N \geq 2^{r}}\left|T_{n}\left(f_{1}-f_{1, N}, f_{2}, \ldots, f_{n}\right)\right|(\lambda)\right\|_{L^{s_{n}}(d \lambda)} \leq C<\infty
$$

for every non-negative integer $r$, with $C$ independent of $r$. Consequently

$$
\sum_{r=0}^{\infty} 2^{n r \delta} \sup _{N \geq 2^{r}}\left|T_{n}\left(f_{1}-f_{1, N}, f_{2}, \ldots, f_{n}\right)\right|(\lambda)<\infty
$$

for almost every $\lambda$. To obtain the conclusion of the lemma, given any $N \geq 1$, choose $r$ so that $2^{r} \leq N<2^{r+1}$ and apply this inequality.

\section{Conclusion of the proof of the main Results}

With the general machinery built up in Sections 4 and 5 in hand, we are now in a position to complete the proofs of our main results. First we prove Theorem 1.3.

Proof. We recall that it suffices to show that under the assumptions of the theorem for a.e. $\lambda \in S$ we can find a function $q(x, \lambda)$ such that $q(x, \lambda) \in C^{1}, q(x, \lambda) \rightarrow 0$, and the condition (9) holds:

$$
\begin{aligned}
q^{\prime}(x, \lambda)- & \frac{i}{2 \operatorname{Im}\left(\theta \bar{\theta}^{\prime}\right)} V(x) \bar{\theta}(x, \lambda)^{2} \exp (-2 i p(x, \lambda)) \\
& \quad-\frac{i}{2 \operatorname{Im}\left(\theta \bar{\theta}^{\prime}\right)} V(x) \theta(x, \lambda)^{2} \exp (2 i p(x, \lambda)) q^{2}(x, \lambda) \in L^{1} .
\end{aligned}
$$

By assumption, the kernels

$$
k_{1}(\lambda, x)=\bar{\theta}(x, \lambda)^{2} \exp (-2 i p(x, \lambda))
$$

and $k_{2}(\lambda, x)=\overline{k_{1}(\lambda, x)}$ satisfy $L^{2}-L^{2}$ and trivial $L^{1}-L^{\infty}$ estimates. Therefore by interpolation, the corresponding operators map $L^{p}$ to $L^{q}$, where $q^{-1}=1-p^{-1}$ and hence $q>p$, for every $1<p<2$. Therefore the theory developed in Sections 4 and 5 applies to the multilinear operators $T_{n}$ from classes $M_{n}$ composed from the kernels $k_{1}(\lambda, x), k_{2}(\lambda, x)$.

We will construct the function $q(x, \lambda)$ by iteration. Let

$$
q^{(0)}(x, \lambda)=-\frac{i}{2 \operatorname{Im}\left(\theta \bar{\theta}^{\prime}\right)} \int_{x}^{\infty} \bar{\theta}(t, \lambda)^{2} \exp (-2 i p(t, \lambda)) V(t) d t
$$


well-defined for almost every $\lambda \in S$ by Theorem 3.1. Given $q^{(n-1)}(x, \lambda)$, we define

$$
\begin{aligned}
q^{(n)}(x, \lambda)= & -\frac{i}{2 \operatorname{Im}\left(\theta \bar{\theta}^{\prime}\right)} \int_{x}^{\infty} \bar{\theta}^{2} \exp (-2 i p) V(t) d t \\
& -\frac{i}{2 \operatorname{Im}\left(\theta \bar{\theta}^{\prime}\right)} \int_{x}^{\infty} \theta^{2} \exp (2 i p) V(t)\left(q^{(n-1)}\right)^{2} d t .
\end{aligned}
$$

Define

$$
V_{x}(y)=V(y) \chi_{\mathbb{R}^{+}}(y-x) .
$$

We need the following

Lemma 6.1. $q^{(n)}(x, \lambda)$ is equal to a sum of multilinear transforms of classes $M_{j}$ (composed from the kernels $k_{1}(\lambda, x)$ and $k_{2}(\lambda, x)$ and with all arguments equal to $\left.V_{x}\right)$ and is defined for almost every $\lambda \in S$. Moreover,

$$
q^{(n)}(x, \lambda)-q^{(n-1)}(x, \lambda)=\sum_{i} T_{j_{i}}\left(V_{x}, V, \ldots, V\right) .
$$

Only finitely many values of orders $j_{i}$ occur in the sum, each satisfying $2 n-1 \leq$ $j_{i} \leq 2^{n+1}-1$.

Proof. We use induction. For $n=0$, all statements are obvious (defining $q^{(-1)}(x, \lambda)$ to be 0$)$. Suppose they are also true for $m \leq n-1$. The fact that $q^{(n)}(x, \lambda)$ is a sum of multilinear transforms of classes $M_{j}$ with some $j$ follows immediately from the induction hypothesis and formula (35). The fact that $q^{(n)}$ is well-defined for a.e. $\lambda \in S$ is then a consequence of Theorem 5.3. Note that

$$
\begin{aligned}
q^{(n)}(x, \lambda)- & q^{(n-1)}(x, \lambda) \\
& =\frac{-i}{2 \operatorname{Im}\left(\theta \bar{\theta}^{\prime}\right)} \int_{x}^{\infty} \theta^{2} \exp (2 i p) V\left(q^{(n-1)}(t, \lambda)-q^{(n-2)}(t, \lambda)\right) \\
\cdot & \left(q^{(n-1)}(t, \lambda)+q^{(n-2)}(t, \lambda)\right) d t .
\end{aligned}
$$

By the induction hypothesis, the right-hand side is a sum of the multilinear transforms from the classes $M_{j}$, where the order $j$ is no less than $(2 n-3)+2=2 n-1$ and no higher than $\left(2^{n}-1\right) 2+1=2^{n+1}-1$.

Now we can complete the proof of Theorem 1.3. If $x^{\epsilon} V(x) \in L^{p}, p \leq 2$, Lemma 5.5 implies that for any multilinear operator of order $j_{i}$

$$
\left|T_{j_{i}}^{\infty, \ldots, \infty}\left(V_{x}, \ldots, V\right)(\lambda)\right| \leq C(\lambda)(1+x)^{-\delta j_{i}}
$$

for almost every $\lambda \in S$ and any $\delta<\epsilon$. Notice that

$$
\begin{aligned}
\left(q^{(n)}\right)^{\prime}(x, \lambda) & -\frac{i}{2 \operatorname{Im}\left(\theta \bar{\theta}^{\prime}\right)} V(x) \bar{\theta}(x, \lambda)^{2} \exp (-2 i p(x, \lambda)) \\
& -\frac{i}{2 \operatorname{Im}\left(\theta \bar{\theta}^{\prime}\right)} V(x) \theta(x, \lambda)^{2} \exp (2 i p(x, \lambda))\left(q^{(n)}\right)^{2}(x, \lambda) \\
= & \frac{i}{2 \operatorname{Im}\left(\theta \bar{\theta}^{\prime}\right)} V(x) \theta(x, \lambda)^{2} \exp (2 i p(x, \lambda))\left(\left(q^{(n)}\right)^{2}(x, \lambda)-\left(q^{(n-1)}\right)^{2}(x, \lambda)\right) .
\end{aligned}
$$


Pick $n$ so that $(2 n-1) \delta>\frac{1}{2}$. Then by Lemmas 6.1 and 5.5 we find that the expression on the right-hand side is absolutely integrable for almost every $\lambda$, since it is the product of some $L^{p}$ function $V(x)$ with a factor which for almost every $\lambda$ is $O\left((1+x)^{-\eta}\right)$ for some $\eta>\frac{1}{2}$, and hence belongs to $L^{q}$ where $q^{-1}=1-p^{-1}$.

Hence $q^{(n)}(x, \lambda)$ satisfies the condition (9) and therefore we can take $q(x, \lambda)=$ $q^{(n)}(x, \lambda)$ for a.e. $\lambda \in S$. Then the first claim of Theorem 1.3 follows from Theorem 2.2 and Lemma 2.1. As a set of full measure in $S$ it suffices to take the set where all multilinear transforms $T_{j}(V, \ldots, V)$ (composed from $k_{1}(\lambda, x), k_{2}(\lambda, x)$ ) of order not larger than $2^{n+1}-1$ converge. It remains to prove the formula (2) for the asymptotic behavior of the eigenfunctions. Notice that the asymptotic behavior stated in Theorem 2.2 differs from the asymptotic behavior we need to show to prove Theorem 1.3 only by the presence of an additional multiplier

$$
\exp \left(\frac{i}{2 \operatorname{Im}\left(\theta \bar{\theta}^{\prime}\right)} \int_{0}^{x}\left(1-|q|^{2}\right)^{-1} V \operatorname{Re}\left(\theta^{2} q \exp (2 i p)\right) d t\right)
$$

in the asymptotic formula for the solutions in Theorem 2.2. But note that the limit of the integral

$$
\int_{0}^{N}\left(1-|q(t, \lambda)|^{2}\right)^{-1} V(t) \operatorname{Re}\left(\theta(t, \lambda)^{2} q(t, \lambda) \exp (-2 i p(t, \lambda))\right) d t
$$

as $N \rightarrow \infty$ exists for a.e. $\lambda$. Indeed, we can expand $\left(1-|q|^{2}\right)^{-1}$ into absolutely convergent series in $|q|^{2}$. Then the whole expression becomes represented as a sum of multilinear transforms $T_{j}^{N, \infty, \ldots, \infty}\left(V_{x}, \ldots, V\right)(\lambda)$. Starting from some $l$, the integrand will become absolutely integrable over the whole axis for a.e. $\lambda$ by Lemma 5.5 ; in the remaining finite sum every term is convergent by Theorem 5.3. Therefore, for a.e. $\lambda$, the expression (36) can be written as $C(\lambda)(1+o(1))$ and hence can be omitted in the asymptotic expression for the solutions. This completes the proof.

We now prove Theorems 1.1 and 1.2.

Proof. It remains only to verify the $L^{2}-L^{2}$ bounds for the corresponding operator (4)). It is convenient to choose $\theta(x, \lambda)=\exp (i \sqrt{\lambda} x)$ in the free case and $\theta(x, \lambda)$ Bloch functions in the periodic case. The corresponding $L^{2}-L^{2}$ bounds were already shown in [14]. For the sake of completeness and since the argument is not very long, we provide here a sketch of the proof for the free case. For the periodic case, the proof is analogous given standard information on the properties of the Bloch functions; see [14] for details.

Without loss of generality, we can restrict our attention to some interval $(a, b)$, $0<a<b<\infty$. Consider $\phi(\lambda) \in C_{0}^{\infty}(0, \infty), 1 \geq \phi(\lambda) \geq 0$, such that $\phi(\lambda)=1$ when $\lambda \in(a, b)$. Clearly it suffices to show $L^{2}-L^{2}$ bounds on functions of compact support for an operator

$$
K f(\lambda)=\phi(\lambda) \int_{0}^{\infty} \exp \left(2 i \sqrt{\lambda} x-\frac{i}{\sqrt{\lambda}} \int_{0}^{x} V(t) d t\right) f(x) d x .
$$


We have

$$
\|K f\|_{L^{2}(a, b)}^{2}=\int_{0}^{\infty} \int_{0}^{\infty} d x d y f(x) f(y) \int d \lambda \phi(\lambda) \exp \left(2 i \sqrt{\lambda}(x-y)-\frac{i}{\sqrt{\lambda}} \int_{y}^{x} V(t) d t\right) .
$$

Let us denote by $Z(x, y)$ the kernel

$$
Z(x, y)=\int d \lambda \phi(\lambda) \exp \left(2 i \sqrt{\lambda}(x-y)-\frac{i}{\sqrt{\lambda}} \int_{y}^{x} V(t) d t\right) .
$$

Let us integrate by parts in $\lambda$ in the expression for $Z(x, y) N$ times, integrating $\exp (2 i \sqrt{\lambda}(x-y))$ and differentiating the rest. We obtain

$$
\begin{aligned}
|Z(x, y)| & \leq C(\phi, a, b, N) \min \left(1,|x-y|^{-N}\right)\left|\int_{y}^{x} V(t) d t\right|^{N} \\
& \leq C(\phi, a, b, N) \min \left(1,|x-y|^{-N\left(1-\frac{1}{q}\right)}\right)\|V\|_{p} .
\end{aligned}
$$

Taking $N$ large enough, for instance such that $N\left(1-\frac{1}{q}\right)>1$, we see that an operator with the kernel $Z(x, y)$ maps $L^{2}$ to $L^{2}$ (by Schur's test, for example). Therefore also

$$
\|K f\|_{L^{2}(a, b)}^{2} \leq C(\phi, a, b)\|f\|_{2}^{2}
$$

We conclude the paper by formulating one simple generalization of Theorem 1.3 (which implies the corresponding generalizations of Theorems 1.1 and 1.2).

Theorem 6.2. Fix a potential $V(x)$. Suppose that there exists a monotone differentiable function $d(x), d(x)>0, d(x) \stackrel{x \rightarrow \infty}{\longrightarrow} 0, d^{\prime}(x) \leq 0$, such that $V(x) d(x)^{-1} \in L^{p}$ with some $p<2$ and $V(x) d(x)^{N} \in L^{1}$ for some integer $N$. Then under the assumptions of Theorem 1.3, all conclusions of Theorem 1.3 hold for the perturbation of $H_{U}$ by $V(x)$.

Proof. Going through the proofs of Lemma 5.5 and Theorem 1.3, we substitute $x^{-\epsilon}$ with $d(x)$. Given that $d(x)^{-1} V(x) \in L^{p}, p<2$, all proofs go unchanged, and in the final step instead of $V(x)(1+x)^{-\delta(2 n-1)}$ being absolutely integrable we need to check that for some $N$,

$$
V(x) d(x)^{N} \in L^{1} .
$$

This is exactly what we assumed in the statement of Theorem 6.2.

We note one additional particular class of potentials which we may treat using Theorem 6.2. Namely, take any $V(x)$ such as in Theorem 1.3, take a sequence $\left\{x_{n}\right\}$ and insert intervals of arbitrary size $I_{n}$ at each point $x_{n}$. Let $\tilde{V}(x)$ be a potential obtained from $V$ by adding such intervals $I_{n}$ where $\tilde{V}$ is zero. Then it is easy to construct a function $d(x)$ with the properties as in Theorem 6.2. For the details of such construction we refer to [13]. 


\section{ApPEndix: Singular POTENTIALS}

In this Appendix, we discuss the preservation of the absolutely continuous spectrum for potentials with strong local singularities. The proof turns out to be almost entirely parallel to the non-singular case, so we mostly sketch the arguments with a few exceptions. We should note, however, that the result has some interest in it. In the explicit construction of the power decaying potentials such that the corresponding Schrödinger operators have purely singular spectrum [24] one can try to use the possible singularity of the potential to get singular spectrum under stronger decay conditions. The results of the appendix show that such a plan does not work out, at least the fundamental exponent $\frac{1}{2}$ and virtually all results we have shown before extend to the situation where strong local singularities are allowed.

We will consider the potentials from the spaces $l^{p}\left(L^{1}\right), 1 \leq p \leq 2$, with the norm given by

$$
\|f\|_{l^{p}\left(L^{1}\right)}=\left(\sum_{n=0}^{\infty}\left(\int_{n}^{n+1}|f(x)| d x\right)^{p}\right)^{\frac{1}{p}} .
$$

The main result we show is

Theorem A.1. Suppose that $U$ is continuous periodic (in particular, the free case $U=0$ is of course included). Let the perturbation $V(x)$ be such that there exist $p \leq 2$ and $\epsilon>0$ with $V(x) x^{-\epsilon} \in l^{p}\left(L^{1}\right)$. Then all conclusions of Theorem 1.2 hold, in particular the absolutely continuous spectrum $S$ of the operator $H_{U}$ is preserved under the perturbation by $V$ and for almost every $\lambda \in S$ there exist solutions of the equation (1) with the pure WKB asymptotic behavior in the main term (i.e. with asymptotic behavior given by (2)).

As before, Theorem A.1 is a corollary of the following general criterion (an analogue of Theorem 1.3):

Theorem A.2. Suppose that the potential $V(x)$ is such that there exist $\epsilon>0$ and $p \leq 2$ so that $x^{\epsilon} V(x) \in l^{p}\left(L^{1}\right)$. Assume that an operator

$$
(K f)(\lambda)=\chi(S) \int_{0}^{\infty} \theta(x, \lambda)^{2} \exp \left(\frac{i}{\operatorname{Im}\left(\theta \bar{\theta}^{\prime}\right)} \int_{0}^{x} V(t)|\theta(t, \lambda)|^{2} d t\right) f(x) d x
$$

satisfies the bound

$$
\|K f(\lambda)\|_{L^{2}(S)} \leq C\|f\|_{l^{2}\left(L^{1}\right)}
$$

on functions $f$ of compact support. Then the absolutely continuous spectrum of $H_{U}$ supported on the set $S$ is preserved under perturbation by $V$, i.e. the set $S$ belongs to the essential support of the absolutely continuous part of the spectral measure of the operator $H_{U+V}$. Moreover, for almost every $\lambda \in S$, there exist solutions $\psi_{\lambda}(x)$, $\bar{\psi}_{\lambda}(x)$ of the equation (1) with the asymptotic behavior (2).

We will indicate the changes in the proof of Theorem 1.3 which are necessary to prove Theorem A.2. First, we need the following substitute for Theorem 3.1.

Lemma A.3. Suppose that $1 \leq p<\infty$, that $q>p$, and that $K$ is a bounded linear operator from $l^{p}\left(L^{1}(\mathbb{R})\right)$ to $L^{q}(\mathbb{R})$. Then the maximal function given by

$$
\left(M_{K} f\right)(\lambda)=\sup _{N}\left|K\left(f \chi_{\mathbb{R}^{+}}(N-x)\right)(\lambda)\right|
$$


satisfies

$$
\left\|M_{K} f\right\|_{q} \leq C_{q}\|f\|_{l^{p}\left(L^{1}\right)}
$$

for the same pair of exponents $p, q$. Moreover the integral defining $\left(M_{K} f\right)(\lambda)$ converges for almost every $\lambda$, for any $f \in l^{p}\left(L^{1}\right)$.

Proof. Given the function $f$ with $\|f\|_{l^{p}\left(L^{1}\right)}=1$, we consider the family of intervals $E(m, l)$ similar to the $L^{p}$ case. Namely, first we consider intervals $E(1,1)$ and $E(1,2)$, such that their union is the whole half-axis, the first interval lies to the left of the second and

$$
\left\|f \chi_{E(1,1)}\right\|_{l^{p}\left(L^{1}\right)}^{p}=\left\|f \chi_{E(1,2)}\right\|_{l^{p}\left(L^{1}\right)}^{p} .
$$

We note that in contrast to the $L^{p}$ case, we cannot in general say that

$$
\left\|f \chi_{E(1, i)}\right\|_{l^{p}\left(L^{1}\right)}^{p}=2^{-1} .
$$

However, it is easy to see that

$$
\left\|f \chi_{E(1, i)}\right\|_{l^{p}\left(L^{1}\right)}^{p} \leq 2^{-1} .
$$

If the sets $E(1,1), E(1,2)$ are unions of the integer intervals $(l, l+1)$, we have equality. Otherwise each set $E(1, i)$ is a union of integer intervals and a set $A_{i}$, such that $A_{1} \cup A_{2}=(m, m+1)$ for some $m$. In this case

$$
\left(\int\left|f \chi_{A_{1}}\right| d x\right)^{p}+\left(\int\left|f \chi_{A_{2}}\right| d x\right)^{p} \leq\left(\int_{m}^{m+1}|f(x)| d x\right)^{p}
$$

(since $p \geq 1$ ), and hence

$$
\left\|f \chi_{E(1,2)}\right\|_{l^{p}\left(L^{1}\right)}^{p}+\left\|f \chi_{E(1,1)}\right\|_{l^{p}\left(L^{1}\right)}^{p} \leq 1 .
$$

We decompose each of the intervals $E(1, i)$ further as in the $L^{p}$ case. In step $m$, we obtain a family of intervals $E(m, l)$, such that $\bigcup_{l} E(m, l)=\mathbb{R}^{+}, E\left(m, l_{1}\right)$ lies to the left of $E\left(m, l_{2}\right)$ if $l_{1} \leq l_{2}$. We choose these intervals so that the total number of the intervals of generation $m$ is $2^{m}$ and $\left\|f \chi_{E(m, l)}\right\|_{l^{p}\left(L^{1}\right)}^{p} \leq 2^{-m}$ for every $l$. Such a family is obtained by splitting every interval of given generation into two equal pieces, with the same arguments as in the first step.

Let

$$
\left(M_{K, m} f\right)(\lambda)=\sup _{l}\left|M_{K}\left(f \chi_{E(m, l)}\right)(\lambda)\right| .
$$

Then

$$
\left(M_{K} f\right)(\lambda) \leq \sum_{m=1}^{\infty}\left(M_{K, m} f\right)(\lambda) .
$$

This follows from the construction of the family $E(m, l)$. Indeed, modulo a set on which $f$ vanishes almost everywhere, any interval $[0, N]$ may be decomposed as a disjoint union of intervals $E(m, l)$, with at most one such interval for each generation number $m$. Summing over $m$ and invoking the triangle inequality leads 
to the desired majorization for $M_{K} f$. Consequently

$$
\left\|M_{K} f\right\|_{q} \leq \sum_{m=1}^{\infty}\left\|M_{K, m} f\right\|_{q}
$$

On the other hand,

$$
\begin{aligned}
\left\|M_{K, m} f\right\|_{q}^{q} & \leq \sum_{l=1}^{2^{m}}\left\|K\left(f \chi_{E(m, l)}\right)\right\|_{q}^{q} \\
& \leq \sum_{l=1}^{2^{m}}\left\|f \chi_{E(m, l)}\right\|_{l^{p}\left(L^{1}\right)}^{q} \leq 2^{m\left(1-\frac{q}{p}\right)} .
\end{aligned}
$$

Therefore,

$$
\left\|M_{K} f\right\|_{q} \leq \sum_{m=1}^{\infty} 2^{m\left(\frac{1}{q}-\frac{1}{p}\right)} \leq C_{q}\|f\|_{l^{p}\left(L^{1}\right)}
$$

(we assumed $\|f\|_{l^{p}\left(L^{1}\right)}=1$, but the bound extends to all $f$ by sublinearity of $M_{K}$ ). Almost everywhere convergence follows from the maximal estimate in a standard way.

Now we prove Theorem A.2.

Proof. The proofs of multilinear transform properties and almost everywhere convergence estimates go exactly the same way as before. The family $E(m, l)$ has the same properties as in the $L^{p}$ case, in particular the martingale-type property (two sets either disjoint or one is contained in another). Lemma 4.3 clearly remains valid. The function

$$
f(x)=\left(\sum_{i=1}^{n}\left|f_{i}(x)\right|^{p}\right)^{\frac{1}{p}}
$$

used to construct the family $E(m, l)$ in the proof, may be replaced by

$$
f(x)=\sum_{i=1}^{n}\left|f_{i}(x)\right| .
$$

The only other change we need to make in the proof is to change $\|\cdot\|_{p}$ to $\|\cdot\|_{l^{p}\left(L^{1}\right)}$ throughout.

To prove Theorem A.1, we need to show the $l^{2}\left(L^{1}\right)-L^{2}(S)$ bounds.

Proof. We will show the proof of the needed norm bound only for the case $U=0$. The general periodic case follows from the properties of the Bloch functions in a way parallel to the free case. We refer to [14] for necessary information and a similar argument.

Clearly we can restrict our attention to some compact interval $I=(a, b), b>$ $a>0$. It is sufficient to show that the operator

$$
(K f)(\lambda)=\phi(\lambda)^{2} \int_{0}^{\infty} \exp \left(2 i \sqrt{\lambda} x-\frac{i}{\sqrt{\lambda}} \int_{0}^{x} V(t) d t\right) f(x) d x
$$

satisfies the $l^{2}\left(L^{1}\right)-L^{2}(I)$ bound on functions of compact support, where $\phi \in$ 
$C_{0}^{\infty}\left(\mathbb{R}^{+}\right)$and $\phi(\lambda)=1$ for $\lambda \in I$. Note that

$$
\begin{aligned}
(K f)(\lambda)= & \sum_{l=0}^{\infty} \phi(\lambda) \exp \left(2 i \sqrt{\lambda} l-\frac{i}{\sqrt{\lambda}} \int_{0}^{l} V(t) d t\right) \\
& \cdot \phi(\lambda) \int_{0}^{1} \exp \left(2 i \sqrt{\lambda} y-\frac{i}{\sqrt{\lambda}} \int_{l}^{y+l} V(t) d t\right) f(y+l) d y .
\end{aligned}
$$

We can write $K f(\lambda)$ as follows:

$$
K f(\lambda)=\sum_{l=0}^{\infty} \phi(\lambda) \exp \left(2 i \sqrt{\lambda} l-\frac{i}{\sqrt{\lambda}} \int_{0}^{l} V(t) d t\right) f(\lambda, l)
$$

where the expression $f(\lambda, l)$ has the following property: for every $m \geq 0$,

$$
\left|\partial_{\lambda}^{m} f(\lambda, l)\right| \leq C_{m}(I, \phi) \sup _{n}\left(\int_{n}^{n+1}|V(x)| d x\right)^{m} f_{l},
$$

where $f_{l}=\int_{l}^{l+1}|f(x)| d x$. This property of $f(\lambda, l)$ is evident from (38).

We compute

$\|K f\|_{L^{2}(I)}^{2} \leq \sum_{l=0}^{\infty} \sum_{m=0}^{\infty} \int d \lambda \phi^{2}(\lambda) \exp \left(2 i \sqrt{\lambda}(l-m)-\frac{i}{\sqrt{\lambda}} \int_{m}^{l} V(t) d t\right) f(\lambda, l) \bar{f}(\lambda, m)$.

Let us integrate by parts, differentiating $f(\lambda, l) \bar{f}(\lambda, m)$ and integrating the rest. By virtually the same computation as one which led us to (37) we obtain

$$
\left|\int d \lambda \phi(\lambda) \exp \left(2 i \sqrt{\lambda}(l-m)-\frac{1}{\sqrt{\lambda}} \int_{m}^{l} V(t) d t\right)\right| \leq C_{r} \min (1,|l-m|)^{-\frac{r}{p}}
$$

for every positive integer $r$. Therefore, taking into account the properties of $f(\lambda, n)$, we obtain

$$
\|K f\|_{L^{2}(I)}^{2} \leq C_{r} \sum_{l=0}^{\infty} \sum_{m=0}^{\infty} \min (1,|m-l|)^{-\frac{r}{p}} f_{m} f_{l}
$$

Taking $r$ large enough $\left(\frac{r}{p}>1\right.$ will do) we see that the operator $K$ satisfies the required bound.

\section{ACKNOWLEDGMENT}

A. Kiselev thanks B. Simon for constant support and encouragement and expresses his gratitude to S. Molchanov and C. Remling for stimulating discussions.

\section{REFERENCES}

[1] P.K. Alsholm and T. Kato, Scattering with long range potentials, Partial Diff. Eq., Proc. Symp. Pure Math. Vol. 23, Amer. Math. Soc., Providence, Rhode Island, 1973, 393-399. MR 52:6215

[2] J. Avron and B. Simon, Transient and recurrent spectrum, J. Funct. Anal. 43(1981), 1-31. MR 83c: 47008

[3] H. Behncke, Absolute continuity of Hamiltonians with von Neumann-Wigner potentials, Proc. Amer. Math. Soc. 111 (1991), 373-384. MR 93c:34160 
[4] M. Ben Artzi and A. Devinatz, Spectral and scattering theory for adiabatic oscillator and related potentials, J. Math. Phys. 20 (1979), 594-607. MR 82a:35088a

[5] V.S. Buslaev and V.B. Matveev, Wave operators for Schrödinger equation with a slowly decreasing potentials, Theor. Math. Phys. 2 (1970), 266-274. MR 57:13246

[6] R. Carmona and J. Lacroix, Spectral Theory of Random Schrödinger Operators, Boston, Birkhauser, 1990. MR 92k:47143

[7] M. Christ and A. Kiselev, A maximal inequality, preprint.

[8] M. Christ, A. Kiselev and C. Remling, The absolutely continuous spectrum of onedimensional Schrödinger operators with decaying potentials, Math. Research Lett., 4 (1997), 719-723. CMP 98:05

[9] E.A. Coddington and N. Levinson, Theory of Ordinary Differential Equations, McGraw-Hill, New York, 1955. MR 16:1022b

[10] W.A. Harris and D.A. Lutz, Asymptotic integration of adiabatic oscillator, J. Math. Anal. Appl. 51 (1975), 76-93. MR 51:6069

[11] D.B. Hinton and J.K. Shaw, Absolutely continuous spectra of second-order differential operators with short and long range potentials, SIAM J. Math. Anal. 17 (1986), 182-196. MR 87c:34038

[12] L. Hörmander, The existence of wave operators in scattering theory, Math. Z. 146 (1976), 69-91. MR 52:14691

[13] A. Kiselev, Absolutely continuous spectrum of one-dimensional Schrödinger operators and Jacobi matrices with slowly decaying potentials, Commun. Math. Phys. 179 (1996), 377-400. MR 97e:81027

[14] A. Kiselev, Stability of the absolutely continuous spectrum of Schrödinger equation under perturbations by slowly decreasing potentials and a.e. convergence of integral operators, Duke Math. J. Vol. 95 (1998), 1-28.

[15] A. Kiselev, Interpolation theorem related to a.e. convergence of integral operators, Proc. Amer. Math. Soc. (to appear). CMP 98:03

[16] A. Kiselev, Y. Last and B. Simon, Modified Prüfer and EFGP transforms and the spectral analysis of one-dimensional Schrödinger operators, Commun. Math. Phys. (to appear).

[17] A. Kiselev, C. Remling and B. Simon, Effective perturbation methods for one-dimensional Schrödinger operators, submitted.

[18] S. Kotani and N. Ushiroya, One-dimensional Schrödinger operators with random decaying potentials, Commun. Math. Phys. 115 (1988), 247-266. MR 89f:60069

[19] V.P. Maslov, S.P. Molchanov, and A.Ya. Gordon, Behavior of generalized eigenfunctions at infinity and the Schrödinger conjecture, Russian J. Math. Phys. 1 (1993), 71-104. MR 95a: 81059

[20] V.B. Matveev, Wave operators and positive eigenvalues for Schrödinger equation with oscillating potential, Theor. Math. Phys. 15 (1973), 574-583.

[21] S. Molchanov, in preparation.

[22] S.N. Naboko, Dense point spectra of Schrödinger and Dirac operators, Theor.-math. 68 (1986), 18-28. MR 88h:81029

[23] M. Reed and B. Simon, Methods of Modern Mathematical Physics, III. Scattering Theory, Academic Press, London-San Diego, 1979. MR 80m:81085

[24] C. Remling, Some Schrödinger operators with power-decaying potentials and pure point spectrum, Commun. Math. Phys. Vol. 186 (1997), 481-493. CMP 97:16

[25] C. Remling, The absolutely continuous spectrum of one-dimensional Schrödinger operators with decaying potentials, Commun. Math. Phys. 4 (1997), no. 5, 719-723. CMP 98:05

[26] B. Simon, Some Schrödinger operators with dense point spectrum, Proc. Amer. Math. Soc. 125(1997), 203-208. MR 97c:34179

[27] B. Simon, Bounded eigenfunctions and absolutely continuous spectra for one-dimensional Schrödinger operators, Proc. Amer. Math. Soc. Vol. 124(1996), 3361-3369. MR 97a:34223

[28] E.M. Stein and G. Weiss, Introduction to Fourier Analysis on Euclidean Spaces, Princeton Univ. Press, Princeton 1971. MR 46:4102

[29] G. Stolz, Bounded solutions and absolute continuity of Sturm-Liouville operators, J. Math. Anal. Appl. 169 (1992), 210-228. MR 93f:34141

[30] J. von Neumann and E.P. Wigner, Über merkwürdige diskrete eigenwerte, Z. Phys. 30(1929), $465-467$. 
[31] J. Weidmann, Spectral Theory of Ordinary Differential Operators, Lecture Notes in Mathematics 1258, Springer-Verlag, Berlin, 1987. MR 89b: 47070

[32] D.A.W. White, Schrödinger operators with rapidly oscillating central potentials, Trans. Amer. Math. Soc. 275 (1983), 641-677. MR 84d:35124

Department of Mathematics, University of California, Berkeley, California 94720

E-mail address: mchrist@math.berkeley.edu

Department of Mathematics, University of Chicago, 5734 University Avenue, Chicago, ILLINOIS 60637

E-mail address: kiselev@math.uchicago.edu 